\title{
Ferric Chelate Reductase 1 Like Protein (FRRS1L) Associates with Dynein Vesicles and Regulates Glutamatergic Synaptic Transmission
}

OPEN ACCESS

Edited by:

Detlev Boison,

Legacy Health, United States

Reviewed by:

Victor Anggono,

The University of Queensland,

Australia

Aleksey V. Zaitsev,

Institute of Evolutionary Physiology and Biochemistry (RAS), Russia

*Correspondence:

Wei LU

luw4@mail.nih.gov

tThese authors have contributed equally to this work.

Received: 01 October 2017 Accepted: 20 November 2017 Published: 08 December 2017

Citation:

Han W, Wang H, Li J, Zhang S and Lu W (2017) Ferric Chelate

Reductase 1 Like Protein (FRRS1L)

Associates with Dynein Vesicles and Regulates Glutamatergic

Synaptic Transmission.

Front. Mol. Neurosci. 10:402. doi: 10.3389/fnmol.2017.00402

\author{
Wenyan Han ${ }^{1 \dagger}$, Huiqing Wang ${ }^{1,2,3+}$, Jun Li ${ }^{1}$, Shizhong Zhang ${ }^{3}$ and Wei Lu ${ }^{1 *}$
}

\begin{abstract}
${ }^{1}$ Synapse and Neural Circuit Research Unit, National Institute of Neurological Disorders and Stroke, National Institutes of Health, Bethesda, MD, United States, ${ }^{2}$ Department of Neurosurgery, The First Affiliated Hospital of Fujian Medical University, Fuzhou, China, ${ }^{3}$ Department of Neurosurgery, Zhujiang Hospital, Southern Medical University, Guangzhou, China
\end{abstract}

In the brain, AMPA receptors (AMPARs)-mediated excitatory synaptic transmission is critically regulated by the receptor auxiliary subunits. Recent proteomic studies have identified that Ferric Chelate Reductase 1 Like protein (FRRS1L), whose mutations in human lead to epilepsy, choreoathetosis, and cognitive deficits, is present in native AMPAR complexes in the brain. Here we have characterized FRRS1L in both heterologous cells and in mouse neurons. We found that FRRS1L interacts with both GluA1 and GluA2 subunits of AMPARs, but does not form dimers/oligomers, in HEK cells. In mouse hippocampal neurons, recombinant FRRS1L at the neuronal surface partially co-localizes with GluA1 and primarily localizes at non-synaptic membranes. In addition, native FRRS1L in hippocampus is localized at dynein, but not kinesin5B, vesicles. Functionally, over-expression of FRRS1L in hippocampal neurons does not change glutamatergic synaptic transmission. In contrast, single-cell knockout (KO) of FRRS1L strongly reduces the expression levels of the GluA1 subunit at the neuronal surface, and significantly decreases AMPAR-mediated synaptic transmission in mouse hippocampal pyramidal neurons. Taken together, these data characterize FRRS1L in heterologous cells and neurons, and reveal an important role of FRRS1L in the regulation of excitatory synaptic strength.

Keywords: FRRS1L, AMPA receptor, C9orf4, auxiliary subunit, dynein

\section{INTRODUCTION}

In the brain, fast excitatory synaptic transmission is largely mediated by glutamate acting on AMPA-type ionotropic glutamate receptors (AMPARs). AMPARs are heterotetrameric assemblies of four subunits, GluA1-4, and the regulation of trafficking and function of AMPARs in neurons underlies many forms of synaptic plasticity, the cellular correlate of learning and memory

Abbreviations: ABHD6, $\alpha / \beta$-Hydrolase domain-containing 6; AMPA, a-amino-3-hydroxy-5-methyl-4-isoxazolepropionic acid; ANOVA, analysis of variance; CKAMP, cysteine-knot AMPAR modulating protein; CNIHs, cornichons; CRISPR, clustered regularly interspaced short palindromic repeats; DIV, days in vitro; EDTA, ethylenediaminetetraacetic acid; EGFP enhanced GFP; EPSC, excitatory postsynaptic currents; FRRS1L, Ferric Chelate Reductase 1 Like protein; GPI, glycophosphatidylinositol; GSG1L, germ cell-specific gene 1-like protein; HA, hemagglutinin; HEK, human embryonic kidney; IP, immunoprecipitation; KO, knock out; NMDA, $N$-methyl-D-aspartate; PNS, post-nuclear supernatant; PNSM, post-nuclear supernatant membrane fractions; PORCN, porcupine; PPR, paired pulse ratio; PSD, postsynaptic density; sgRNA, single-guide RNA; TARP, transmembrane AMPA receptor regulatory proteins; vGluT, vesicular glutamate transporter 1 ; WT, wild-type. 
(Malinow and Malenka, 2002; Bredt and Nicoll, 2003; Malenka and Bear, 2004; Anggono and Huganir, 2012; Huganir and Nicoll, 2013). Accumulating studies over the last decade have shown that trafficking and function of AMPARs are critically controlled by an ever-growing list of membrane proteins, including TARPs (Chen et al., 2000; Tomita et al., 2003; Jackson and Nicoll, 2011), CNIHs (Schwenk et al., 2009), SynDig1 (Kalashnikova et al., 2010), CKAMPs/SHISAs (von Engelhardt et al., 2010; Farrow et al., 2015; Klaassen et al., 2016), GSG1L (Schwenk et al., 2012; Shanks et al., 2012; McGee et al., 2015; Gu et al., 2016a), PORCN (Erlenhardt et al., 2016), $\alpha / \beta$-ABHD6 (Erlenhardt et al., 2016; Wei et al., 2016) and carnitine palmitoyltransferase 1C (CPT1C) (Gratacos-Batlle et al., 2014; Fado et al., 2015). These membrane proteins regulate AMPAR biogenesis, trafficking, gating, and pharmacological properties, and often play overlapping, but also distinct, roles in the regulation of AMPAR-mediated synaptic transmission (Jackson and Nicoll, 2011; Straub and Tomita, 2012; Haering et al., 2014; Greger et al., 2017; Jacobi and von Engelhardt, 2017).

Recent proteomic studies have identified several uncharacterized proteins, including transmembrane, GPIanchored and secreted molecules, that are associated with endogenous AMPARs in the brain (von Engelhardt et al., 2010; Schwenk et al., 2012; Chen et al., 2014). Understanding the function of these molecules in the regulation of AMPARs will be important for elucidation of the regulatory mechanisms for controlling excitatory synaptic transmission. One of these proteins is FRRS1L, which is also named C9orf4 (Schwenk et al., 2012). Human and mouse genetic studies have shown a critical role of FRRS1L in human and animal behavior (Madeo et al., 2016; Shaheen et al., 2016). Indeed, loss-of-function mutations of FRRS1L in human lead to epilepsy, prominent choreoathetosis, and severe impairment of cognitive functions (Madeo et al., 2016; Shaheen et al., 2016). Thus, it is important to understand the role of FRRS1L in the regulation of synaptic transmission.

Here we have performed basic characterizations of FRRS1L in heterologous cells and in neurons, and have employed gain-offunction and loss-of-function approaches to examine the role of FRRS1L in the regulation of glutamatergic synaptic transmission. We find that over-expressed FRRS1L in hippocampal neurons only co-localizes with a portion of the AMPAR GluA1 subunit at the plasma membrane, suggesting that FRRS1L might be involved in the regulation of a subpopulation of AMPARs in neurons. Interestingly, native FRRS1L is localized at dynein, but not kinesin KIF5B, vesicles. Functionally, FRRS1L does not regulate AMPAR abundance at the cell surface in HEK cells, and similarly, over-expression of FRRS1L in culture hippocampal neurons does not change the strength of glutamatergic synaptic transmission. In contrast, single guide RNA (sgRNA)-based single-cell knockout (KO) of FRRS1L in hippocampal neurons leads to a modest, but significant, reduction of AMPAR-mediated synaptic transmission. Importantly, the sgRNA-mediated effect can be fully rescued by a sgRNA resistant FRRS1L construct in neurons. Taken together, these data reveal a distinct subcellular distribution of FRRS1L in hippocampal neurons and demonstrate an important role of FRRS1L in the regulation of AMPAR-mediated synaptic transmission in hippocampus.

\section{MATERIALS AND METHODS}

\section{Mouse Genetics}

This study was carried out in accordance with the recommendations of the Animal Care and Use Committee (ACUC) guidelines at National Institute of Neurological Disorders and Stroke (NINDS), National Institutes of Health (NIH). The animal protocols were approved by the NINDS ACUC at NIH (protocol number: 1339-15). Mice of either sex were used. Animals were given access to food and water ad libitum.

\section{Plasmids}

The plasmid encoding mouse FRRS1L was purchased from Origene (Cat. MC212878). pCAGGS/FRRS1L-IRES-EGFP plasmid was generated by inserting FRRS1L coding sequence to pCAGGS-IRES-EGFP or pCAGGS-IRES-mCherry vector. $\mathrm{N}$-terminal human influenza hemagglutinin (HA) tag was inserted after the signal peptide sequence of FRRS1L (HAFRRS1L) through an overlapping PCR strategy and the amplicon was ligated in pcDNA3.0 vector (Invitrogen). C-terminal myc tag was added through the standard PCR strategy and ligated in pcDNA3.0 vector for FRRS1L-myc plasmid. Flag-GluA1 and Flag-GluA2 (Q) plasmids were gifts from Dr. Katherine Roche's lab at NINDS, NIH. The pspCas9(bb)-2A-IRES-GFP plasmid was purchased from Addgene (Cat. 48138) for sgRNA cloning. FRRS1L sgRNAs were generated by a general PCR procedure with the following sgRNA sequences (accession number: NP_001136437, \#1: gcgggatccgtgcgcagcgatgg; \#2: ccccgcggacgacagcgcgggcc; \#3: accccggggacgcgcgcgagggg). FRRS1L sgRNAs were inserted into pspCas9(bb)-2A-IRES-GFP vector at the BbsI cutting site. The underlined sequence of sgRNA $^{\# 3}$ was the mutation region for the $\operatorname{sgRNA}^{\# 3}$ resistant plasmid, FRRS1L* (mutation region: accccggggacgcgcgcgagggg to acccagaggcaggagcaagagggg; amino acids: Arg-Gly-Arg-Ala-Arg) (template: FRRS1L-myc in pcDNA3.0 vector), which were inserted into pCAGGS-IRES-mCherry vector. All plasmids were confirmed by standard DNA sequencing.

\section{Co-immunoprecipitation and Western Blot in HEK Cells}

For co-immunoprecipitation (Co-IP) experiments, FlagGluA1, Flag-GluA2 and HA-FRRS1L constructs were used for transfection in HEK cells. HEK cells were cultured and transfected with effectene transfection reagent (Qiagen, Cat. 301425) as described previously ( $\mathrm{Gu}$ et al., 2016a). Forty-eight hours after transfection, cells were homogenized in ice-cold lysis buffer containing $25 \mathrm{mM}$ Tris (pH 7.4), 1\% Triton X-100, $150 \mathrm{mM} \mathrm{NaCl}, 5 \%$ glycerol, $1 \mathrm{mM}$ EDTA, and EDTA-free protease inhibitors (Roche, 5892791001). Equal amounts of cell lysates were incubated with anti-Flag M2 affinity beads (Sigma, A2220) overnight at $4^{\circ} \mathrm{C}$. Beads were washed three times with $300 \mu l$ lysis buffer and diluted in equal amount of $2 \times$ loading buffer (Bio-Rad 161-0737) containing 10\% $\beta$-mercaptoethanol (BME) (Fisher Scientific BP176100). Samples were then resolved by SDS-PAGE with $10 \%$ precast gel (Bio-Rad 
4561083) and were subjected to immunoblotting with anti-HA (rabbit, 1:1,000, Santa Cruz, sc-805) or anti-Flag (rabbit, 1:1,000, Sigma F2555) antibodies. For the dimerization experiment, HA-FRRS1L and FRRS1L-Myc plasmids were co-transfected into HEK cells for $48 \mathrm{~h}$. Anti-HA and anti-Myc (rabbit, 1:1,000, Cell signaling, 2278S) antibodies were used in this experiment. For FRRS1L sgRNA screening experiment, FRRS1L-Myc and sgRNA candidates were co-transfected in a ratio at 1:2 to HEK cells $\left(2 \times 10^{6}\right.$ cells/well on transfection day in 6 -well plate), while empty pcDNA3.0 vector was added in FRRS1L-Myc transfected cells to balance the total amount of DNA. All data were collected from at least three independent experiments.

\section{Mouse Tissue Preparations and Western Blotting}

Mouse tissues of interest (6-8-week old for tissue distribution experiments) were quickly dissected after anesthetizing the donor mice with isoflurane (Baxter, NDC10019-360-40), then placed in $2.0 \mathrm{ml}$ microfuge tubes and kept in dry ice to snap freeze. The samples were separately added into an ice-cold glass pestle containing $1 \mathrm{ml}$ ice-cold non-detergent homogenization buffer [250 mM sucrose, $1 \mathrm{mM}$ EDTA, $10 \mathrm{mM}$ Tris- $\mathrm{HCl}$ buffer ( $\mathrm{pH}$ 7.2), and EDTA-free protease inhibitors (1 tablet/10 ml, Roche, 5892791001)] to homogenize. The homogenate was then sonicated with two 10 -s pulses (30 s pause in between pulses) using an ice-cold ultrasonic probe, and then centrifuged at $1,000 \times g$ for $10 \mathrm{~min}$ to remove neuronal nuclei and other cell debris. The crude post-nuclear supernatant fraction (PNS) was transferred to a $1.5 \mathrm{ml}$ ultracentrifuge tube; the pellet containing the nuclei was discarded. The PNS was centrifuged at $100,000 \times g$ for $2 \mathrm{~h}$ at $4^{\circ} \mathrm{C}$ to collect membrane fractions in the pellet. The membrane fractions were lysed in appropriate volume of ice-cold lysis buffer containing $25 \mathrm{mM}$ Tris ( $\mathrm{pH} 7.4$ ), 1\% Triton X-100, $150 \mathrm{mM} \mathrm{NaCl}, 5 \%$ glycerol, $1 \mathrm{mM}$ EDTA, and EDTA-free protease inhibitors. Equal amounts of lysates measured by BCA protein assay kit (Thermofisher, Cat. 23225) were subjected to SDS-PAGE and immunoblotting with an anti-FRRS1L (1:1,000, Santa Cruz, sc-398692) or anti- $\alpha$-tubulin antibody (1:5000, Sigma, T8203). All data were collected from at least three independent experiments.

\section{Vesicle Immunoisolation}

Adult WT mouse hippocampal tissue (6-8-week old) was homogenized in ice-cold $500 \mu \mathrm{l}(20 \mathrm{mg}$ tissue/100 $\mu \mathrm{l})$ detergent-

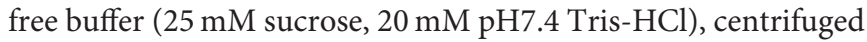
at $1,000 \times g$ for $10 \mathrm{~min}$ at $4^{\circ} \mathrm{C}$ (Encalada et al., 2011). The buffer contained an EDTA-free protease inhibitor (Roche, 5892791001). The $400 \mu l$ hippocampal homogenate was bottom-loaded on a sucrose step gradient consisting of 35\% (400 $\mu \mathrm{l})$, and $8 \%(600 \mu \mathrm{l})$ sucrose in each $1.5 \mathrm{ml}$ centrifuge tube (VMR 16466-064). After centrifugation at $200,000 \times \mathrm{g}$ for $2 \mathrm{~h}$ at $4^{\circ} \mathrm{C}$, the $8 / 35,35 / 40$, and post-ultracentrifuge PNS membrane interphases (PNSM) were separately harvested and incubated with Dynein (1:300, MAB1618, Millipore), Kinesin (1:300, MAB1614, Millipore) or IgG (1:300, Sigma) antibodies overnight. The next day, washed $50 \mu$ l protein G dynabeads (ThermoFisher, 10007D) were added into the antibody-incubated fractions followed by incubating with rotation for $1 \mathrm{hr}$ at RT. Washed pellets on magnet were eluted with $20 \mu \mathrm{l}$ elution buffer. Eluted sample was mixed with $2 \times$ loading buffer containing $10 \% \mathrm{BME}$ and heated at $60^{\circ} \mathrm{C}$ for $10 \mathrm{~min}$. The 8/35, 35/40 and post-ultracentrifuge PNSM were analyzed by SDS-PAGE and immunoblotted by anti- FRRS1L (1:1,000), $\gamma 8$ (1:1,000, rabbit, SigmaMillipore, Cat. AB9876) or CNIH2 (1:1,000, rabbit, Synaptic Systems, Cat. 253203) antibodies. The $8 / 35$ interphase incubated with dynein/kinesin was also immunoblotted by anti-AMPAR (PAN) $(1: 1,000$, mouse, SigmaMillipore, MABN832) and dynein/kinesin antibodies.

\section{Immunocytochemistry in HEK Cells}

For surface and total AMPAR subunit expression, HEK cells $\left(2 \times 10^{4}\right.$ cells/coverslip) cultured on coverslips were transfected with Flag-GluA1 or Flag-GluA2 on their own or together with pCAGGS/FRRS1L-IRES-mCherry by effectene transfection reagent, and incubated in $37^{\circ} \mathrm{C}$ incubator containing $5 \% \mathrm{CO}_{2}$ for approximate $40-48 \mathrm{~h}$. After removing the cell media, cells were washed once by $1 \times$ PBS and then fixed with ice-cold fixation buffer (10 $\mathrm{ml} \mathrm{16 \%} \mathrm{paraformaldehyde} \mathrm{solution,} 8 \mathrm{ml} 5 \times$ PBS and $1.6 \mathrm{~g}$ sucrose dissolved in $22 \mathrm{ml}$ distilled water) for $15 \mathrm{~min}$. Cells were then blocked by PBS containing 10\% normal goat serum (NGS) (Vector Laboratories) for $30 \mathrm{~min}$. Without permeabilization, cells were incubated with a monoclonal anti-Flag antibody $(1: 1,000)$ dissolved in $1 \times$ PBS containing 3\% NGS at room temperature (RT) for $2 \mathrm{~h}$ for surface Flag (sFlag) tag labeling. After three times washing by $1 \mathrm{x}$ PBS, cells were permeabilized with $0.2 \%$ Triton X-100 for $15 \mathrm{~min}$, and then blocked for $30 \mathrm{~min}$ in $1 \times$ PBS containing $10 \%$ NGS. Cells were then incubated with a polyclonal anti-Flag antibody $(1: 1,000)$ at $4^{\circ} \mathrm{C}$ overnight for total Flag (tFlag) tag labeling. Alexa Fluor (AF) 647 anti-mouse and AF488 anti-rabbit secondary antibodies (Molecular Probe) were used to label sFlag and tFlag. For co-localization of surface and total HA-FRRS1L or sFlag- GluA1/GluA2 in HEK cells, the cells were transfected and treated as above with indicated plasmids, and were subjected to similar immunolabeling procedures as described above. Coverslips were mounted with Fluoromount G (Southern Biotech) for imaging acquisition.

\section{Hippocampal Primary Dissociated Neuronal Culture}

Hippocampal primary neuron cultures were prepared as described before ( $\mathrm{Gu}$ et al., 2016b). Briefly, hippocampi from $\sim$ E18 C57B6 mouse embryos were microdissected in ice-cold dissection media (49 $\mathrm{ml} \mathrm{Ca}^{2+} / \mathrm{Mg}^{2+}$ free Hanks media, $0.5 \mathrm{ml}$

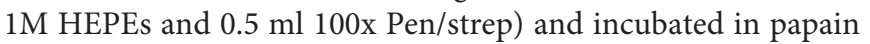
solution (papain $\left(110 \mathrm{u} /\right.$ vial) dissolved in $5 \mathrm{ml} \mathrm{Ca}{ }^{2+} / \mathrm{Mg}^{2+}$ free Hanks media and $250 \mu \mathrm{l}$ DNase-I $(1100 \mathrm{u} / \mathrm{vial})$ for $45 \mathrm{~min}$ followed by centrifuging at $800 \mathrm{rpm}$ for $5 \mathrm{~min}$. The pellet was resuspended in Hanks solution (Invitrogen 14025-092) containing DNase-I (Worthington 3170) and dissociated into single cells by gentle pipette trituration. Digestion was then stopped by adding trypsin inhibitor $(10 \mathrm{mg} / \mathrm{ml}$, Sigma T9253) and BSA $(10 \mathrm{mg} / \mathrm{ml}$, Sigma A9647), and centrifuged at $800 \mathrm{rpm}$ for $10 \mathrm{~min}$. The pellet was re-suspended in neurobasal plating media containing $2 \%$ 
FBS, 2\% B27 supplements, and L-glutamine (2 mM). Neurons were plated at a density of $\sim 1.5 \times 10^{5}$ cells/coverslip on coverslips pretreated with poly-D-lysine (Sigma P7886) in a 24-well plate. Culture media were replaced by half once per week.

\section{Immunocytochemistry in Neurons}

Mouse hippocampal cultured neurons were transfected with indicated FRRS1L sgRNA ${ }^{\# 1}$ or sgRNA $^{\# 3}$ plasmids by calcium phosphate transfection at DIV2 as described (Li et al., 2017). Endogenous GluA1 in live neurons was labeled with a mouse monoclonal antibody against an extracellular epitope of GluA1 (clone $\mathrm{RH} 95 ; 5 \mu \mathrm{g} / \mathrm{ml}$ ) in conditioned culture medium for $15 \mathrm{~min}$ at $37^{\circ} \mathrm{C}$ at DIV16. After being washed by $1 \times \mathrm{PBS}$, neurons were fixed with ice-cold fixation buffer for $15 \mathrm{~min}$. After permeabilization using $0.2 \%$ Triton $\mathrm{X}-100$ in $1 \times \mathrm{PBS}$ for $15 \mathrm{~min}$ at RT, neurons were blocked for $1 \mathrm{~h}$ in $1 \times \mathrm{PBS}$ containing 5\% NGS. Cells were then incubated with anti-GluA1 (SigmaMillipore, AB1504, 1:1,000) antibody at $4^{\circ} \mathrm{C}$ overnight followed by incubation with AF-647 (tGluA1) and AF-555 secondary antibodies (sGluA1, Molecular Probe). In FRRS1L overexpression experiments, mouse hippocampal dissociated neurons were transfected with HA-FRRS1L plasmid by DNAIn neuro transfection reagent (MTI-GlobalStem, Cat. GST-2101) at DIV10. Cells at DIV14-16 were immunostained as described above. For co-localization of surface HA-FRRS1L (sHA-FRRS1L) and surface endogenous GluA1, GluA2 or PSD95 (anti-PSD95, Sigma Millipore, AB9708), neurons were transfected with HAFRRS1L by Neuro-In DNA transfection reagent at DIV10 and fixed on DIV16.

\section{Image Acquisition}

For immunostaining in HEK cells, images were acquired on a Zeiss LSM 880 laser scanning confocal microscope using a $63 \mathrm{x}$ oil objective (1.4 numerical aperture). Multiple $z$ sections (9 optical slices) were acquired at $0.5-1.0 \mu \mathrm{m}$ intervals. Images were captured using a $1024 \times 1024$ pixel screen, and gains for both fluorophores were set between 700 and 800 . For immunostaining in neuronal cultures, images were acquired on a Zeiss LSM 880 laser scanning confocal microscope using a $63 \mathrm{x}$ oil objective (1.4 numerical aperture). Image acquisition was performed with identical settings for a specific experiment. Multiple $\mathrm{z}$ sections of secondary apical dendrites were collected at 1.0-1.5 $\mu \mathrm{m}$ intervals with $512 \times 512$ pixel screen. Pinhole was set to 1 airy unit for all experiments. Scan speed function was set to 9 and the mean of 4 lines was applied. Laser power, digital gain, and offset settings were all identical in each experiment by using the "reuse" function in LSM software.

\section{Images Analysis}

For quantitative analysis of immunostaining in HEK cells and in neurons, maximal projection images were created with ZEN software (Zeiss) from 4 to 6 serial optical sections. For each image collected containing sGluA1 or PSD95, dendritic outline was drawn to cover $20-30 \mu \mathrm{m}$ in length (representing a surface area of 850-1,000 pixels). The integrated fluorescent intensity targeting protein was calculated from one segment of dendrite positive for HA-FRRS1L. Using Metamorph (Universal
Imaging Corp.), quantitation of the fluorescence signal from sFlag or tFlag in HEK cells and surface/total AMPAR subunits in neurons were determined from fluorescent signal above a threshold. The threshold value was held identical within single experiments, and only slightly adjusted between independent experiments. For surface/total ratio of GluA1 staining and GFP fluorescent signals in dissociated hippocampal neuronal cultures, maximal projection images were generated by the LSM880 Brower software. Background was subtracted by using the "subtract background" function in Image J software (NIH), and the background level was held identical for all cells within each experiment. Region of interest (ROI) was defined along a segment of the dendrite 20-30 $\mu \mathrm{m}$ according to the fluorescence signal distinguished from the background in ImageJ software. Average values of fluorescence intensities in ROI (the total fluorescent intensity divided by the total area of a dendritic segment) were calculated by ImageJ.

For co-localization analysis in cultured hippocampal neurons, 25-30 $\mu \mathrm{m}$ secondary dendritic segments were chosen as ROI. HA-FRRS1L, GluA1 or PSD-95 puncta were thresholded and confirmed visually to select appropriate clusters following a minimal size cut-off, which included all recognizable clusters. The co-localization percentage was quantified by the measurement of HA-FRRS1L-positive GluA1 or PSD-95 puncta divided by total number of thresholded GluA1 or PSD-95 puncta. 10-27 dendrites were analyzed from approximately 5-10 neurons.

\section{In Situ Hybridization (ISH) in Cultured Neurons}

Cultured neurons on coverslips were transfected with sgRNAs at DIV2 and fixed in fixation buffer at RT for $15 \mathrm{~min}$ at DIV16. Coverslips were then washed three times with $1 \times$ PBS and sequentially incubated in 50,70, and $100 \% \mathrm{EtOH}$ for $5 \mathrm{~min}$ for dehydration. The coverslips were then submerged in 70 and $50 \% \mathrm{EtOH}$ for $2 \mathrm{~min}$ for rehydration followed by keeping in $1 \times$ PBS for $10 \mathrm{~min}$. The coverslips were then treated with hydrogen peroxide and protease III (1:15 diluted in $1 \times$ PBS) (RNAscope, 2000258) for $10 \mathrm{~min}$ followed by washing twice in $1 \times$ PBS. The coverslips were quickly transferred to the barriered SuperFrost Plus slide. All of the above steps were operated at RT. Four drops $(\sim 50-80 \mu \mathrm{l})$ of FRRS1L probe (RNAscope, 16137A) were added to submerge the coverslip, then the slide was incubated in a humidity control tray at $40^{\circ} \mathrm{C}$ for $2 \mathrm{~h}$ followed by washing twice in $1 \times$ wash buffer (RNAscope, 2000003) for $2 \mathrm{~min}$ at RT. The slide was then respectively incubated with detection reagents AMP1-6 and Fast RED reagent (A:B, 1:60 ratio) (RNAscope, 2000173) as described in RNAscope HD 2.5 RED reagent protocol (322360USM). After rinsing in $1 \mathrm{x}$ wash buffer, the slide was removed from the humidity control tray and dried in dry oven at $60^{\circ} \mathrm{C}$ for $20 \mathrm{~min}$. The slide containing coverslip was then dipped in fresh Xylene and 3 drops of DAPI permount media were quickly added followed by covering with a $24 \mathrm{~mm} \times 50 \mathrm{~mm}$ coverslip without air bubbles.

Positive punctate signal was detected under a standard bright field microscope at 63x magnification using confocal microscope. GFP positive signals and red punctate dots were presented 
in FITC and $550 \mathrm{~nm}$ channels. The staining of ISH was categorized into five grades based on the recommendation from the RNAscope manufacturer (322360-USM): $0,1+, 2+, 3+$, and $4+$. Staining Scores were defined by the following criteria: 0 , no staining or less than 1 dot per 10 cells; $1,1-3$ dots per cell; $2,4-10$ dots per cell, very few dot clusters; $3,>10$ dots per cell, less than $10 \%$ positive cells have dot clusters; $4,>10$ dots per cell, more than $10 \%$ positive cells have dot clusters.

\section{In Utero Electroporation}

Timed-pregnant WT C57B6 mice at embryonic 14.5 days (E14.5) were anesthetized with isoflurane. The abdominal cavity was opened and 7-9 embryos in the uterine horns were gently exposed. The lateral ventricle of each embryo was manually injected with approximately 1-2 $\mu$ l of FRRS1L sgRNA plasmid at a concentration of $2 \mu \mathrm{g} / \mu \mathrm{l}$ mixed with $0.05 \%$ fast green. The pipettes were beveled with a BV-10 micropipette Beveller (Sutter) before microinjection. After unilateral injection in embryos, voltage steps via tweezertrodes $(5 \mathrm{~mm}$ round, platinum electrodes and BTX electroporator, BTX, ECM830) positioned on either side of the embryo head were applied across the uterus to target hippocampal neural progenitors. Voltage was set at $45 \mathrm{~V}$ for 5 pulses at $1 \mathrm{~Hz}$, each pulse lasting $50 \mathrm{~ms}$. The embryos were then moistened with sterilized PBS (pre-warmed at $37^{\circ} \mathrm{C}$ ) and returned to the abdominal cavity. Buprenex $(0.1 \mathrm{mg} / \mathrm{kg})$ was then applied and the wound was subsequently sutured.

\section{Electrophysiology in Hippocampal Slices}

Organotypic hippocampal slice cultures were prepared and transfected as previously described ( $\mathrm{Gu}$ et al., 2016a). Both sexes of C57B6 mice at the age of p6-p8 were used for organotypic hippocampal slice cultures. About 2-4 days after culture, slices were transfected with FRRS1L-IRES-EGFP or with FRRS1L sgRNA ${ }^{\# 1}$ or sgRNA ${ }^{\# 3}$ plasmids by gene gun-mediated transfection. Slices were cultured for an additional 2-5 days for over-expression or 14-16 days for sgRNA transfection. After transfection, slices were transferred to a submersion chamber on an upright Olympus microscope, perfused in normal ACSF containing (in mM) $\mathrm{NaCl} 119, \mathrm{KCl} 2.5, \mathrm{NaHCO}_{3}$ 26.2, $\mathrm{NaH}_{2} \mathrm{PO}_{4}$ 1, glucose $11, \mathrm{CaCl}_{2}$ 4.0, and $\mathrm{MgSO}_{4}$ 4.0, with picrotoxin $(100 \mu \mathrm{M})$, and saturated with $95 \% \mathrm{O}_{2} / 5 \% \mathrm{CO}_{2}$. For recording evoked EPSCs in organotypic slices, ACSF was also supplemented with 5-20 $\mu \mathrm{M}$ 2-chloroadenosine to dampen epileptiform activity. EGFP-positive neurons in organotypic slice cultures were identified by epifluorescence microscopy. For acute hippocampal slices, mouse pups at p14-p16 after embryonic IUE were euthanized by decapitation. The brain was immediately placed in ice-cold cutting solution containing (in $\mathrm{mM}) \mathrm{KCl} 2.5, \mathrm{CaCl}_{2}$ 0.5, $\mathrm{MgCl}_{2} 7, \mathrm{NaH}_{2} \mathrm{PO}_{4} \quad 1.25, \mathrm{NaHCO}_{3}$ 25 , glucose 7 , ascorbic acid 1.3 and sucrose 210 as previously described ( $\mathrm{Gu}$ et al., 2016b). The hippocampi were quickly dissected out on an ice-cold platform and immediately placed onto an ice-cold Argrose gel block (5\% Argrose). The gel block was quickly glued on the cutting platform containing ice-cold cutting solution saturated with carbogen. $300 \mu \mathrm{m}$ transverse slices were cut and recovered at $32^{\circ} \mathrm{C}$ for $30-60 \mathrm{~min}$. Slices were then maintained in ACSF (modified to containing
$2.5 \mathrm{mM} \mathrm{CaCl}_{2}$ and $1.3 \mathrm{mM} \mathrm{MgCl}_{2}$ ) at RT for 30-60 min prior to recording. The slice was then transferred to a recording chamber that is mounted on an upright Olympus microscope. The recording chamber was continuously perfused with ACSF containing picrotoxin $(100 \mu \mathrm{M})$ and saturated with $95 \% \mathrm{O}_{2} / 5 \%$ $\mathrm{CO}_{2}$.

All paired recordings involved simultaneous whole cell recordings from one EGFP-positive neuron and a neighboring EGFP-negative control neuron. Cells were recorded with 3to 5- MO borosilicate glass pipettes. The internal solution contained $\mathrm{CsMeSO}_{4} 135 \mathrm{mM}, \mathrm{NaCl} 8 \mathrm{mM}$, HEPEs $10 \mathrm{mM}$, $\mathrm{Na}_{3}$ GTP $0.3 \mathrm{mM}$, MgATP $4 \mathrm{mM}$, EGTA $0.3 \mathrm{mM}$, QX-314 $5 \mathrm{mM}$ and spermine $0.1 \mathrm{mM}$. The stimulus was adjusted to evoke a measurable, monosynaptic EPSC in both cells. AMPA EPSCs were measured at a holding potential of -70 $\mathrm{mV}$, and NMDA EPSCs were measured at $+40 \mathrm{mV}$ and at $100 \mathrm{~ms}$ after the stimulus, at which point the AMPA EPSC has completely decayed. Paired-pulse ratios (PPRs) were measured by giving two pulses at a $50-\mathrm{ms}$ interval and taking the ratio of the two peaks of the EPSCs from an average of 30-50 sweeps. Series resistance was monitored and not compensated, and cells in which series resistance varied by $25 \%$ during a recording session were discarded. Synaptic responses were collected with a Multiclamp 700B amplifier (Axon Instruments, Foster City, CA, United States), filtered at $2 \mathrm{kHz}$ and digitized at $10 \mathrm{kHz}$. All pharmacological reagents were purchased from Abcam, and other chemicals were purchased from Sigma.

In the scatter plots for simultaneous dual recordings (Figures 4, 6), each open circle represents one paired recording, and the closed circle represents the average of all paired recordings. In the scatter plot, the $\mathrm{x}$-axis represents the EPSC recorded in the control cell, and the $y$-axis represents the EPSC recorded in the transfected cell. Virtual 1:1 diagonal line is also shown. If the data point falls above the diagonal line, it indicates that the EPSC is higher in the transfected cell. If it falls below the diagonal line, it indicates that the EPSC is higher in the control cell.

For decay time constants of AMPA EPSCs, a single weighted decay time constant was calculated from the area under the peaknormalized current (Cathala et al., 2005), according to

$$
\mathrm{t}_{\text {decay }}=\frac{1}{\mathrm{l}_{\text {peak }}} \int_{\mathrm{t}_{\text {peak }}}^{\mathrm{t}_{0}} 1(\mathrm{t}) \mathrm{dt},
$$

where $t_{0}$ was $80 \mathrm{~ms}$ after the peak.

\section{Glutamate Puffing Experiments in HEK Cells}

For glutamate-evoked whole-cell currents in HEK cells, cells $\left(2 \times 10^{4}\right.$ cells/coverslip) were cultured and transfected with GluA1, GluA2, GluA1 plus FRRS1L-IRES-GFP (ratio at 1:1), or GluA2 plus FRRS1L-IRES-GFP (ratio at 1:1) (an empty plasmid was used to make the total cDNA amount, $0.4 \mu \mathrm{g}$, per transfection). On the day of recording, coverslips with transfected HEK cells were transferred to a submersion chamber on an upright Olympus microscope perfused with external solution 
containing (in $\mathrm{mM}$ ) $\mathrm{NaCl} 140, \mathrm{KCl} 5, \mathrm{MgCl}_{2}$ 1.4, EGTA 5, HEPES 10, $\mathrm{NaH}_{2} \mathrm{PO}_{4}$ 1, D-glucose 10, and NBQX 0.01, with $\mathrm{pH}$ adjusted to 7.4. The internal solution contained $\mathrm{CsMeSO}_{4}$ $135 \mathrm{mM}$, NaCl $8 \mathrm{mM}$, HEPEs $10 \mathrm{mM}$, Na ${ }_{3} \mathrm{GTP} 0.3 \mathrm{mM}$, MgATP $4 \mathrm{mM}$, EGTA $0.3 \mathrm{mM}$, QX-314 $5 \mathrm{mM}$, and spermine $0.1 \mathrm{mM}$. Glutamate induced whole-cell currents in HEK cells were recorded at $-70 \mathrm{mV}$ by local fast application (0.5 s) of $1 \mathrm{mM}$ glutamate and $100 \mu \mathrm{M}$ cyclothiazide, dissolved in the external solution. The tip of Mini-manifold was placed at $100 \mu \mathrm{m}$ away from the recorded HEK cells. Whole-cell currents were collected with a Multiclamp 700B amplifier (Axon Instruments, Foster City, CA, United States), filtered at $2 \mathrm{kHz}$ and digitized at $10 \mathrm{kHz}$. All pharmacological reagents were purchased from Abcam, and other chemicals were purchased from Sigma.

\section{Statistical Analysis}

Statistical analysis was performed using GraphPad Prism 7. Comparisons between two groups were performed with twotailed, unpaired $t$-tests except for paired recording whereby the statistic comparisons were performed with two-tailed, paired $t$-test. Multiple comparisons with three or more groups were performed with one-way ANOVAs. The D'Agostino and Pearson normality test and the Shapiro-Wilk normality test were performed to test the normality of unpaired data. All of the data passed the normality test $(\alpha=0.05)$ and came from a Gaussian distribution $(p>0.05)$. Significance was considered when the $p$-value $<0.05,0.01,0.001$ or 0.0001 (indicated as $*, * *, * * *$, or ${ }^{* * * *}$, respectively). $p$-values $\geq 0.05$ were considered not significant. The data were presented as Mean \pm SEM.

\section{RESULTS}

\section{Basic Characterizations of FRRS1L in Mouse}

Recent proteomic studies have identified FRRS1L as a component of native AMPAR complexes in the brain (Schwenk et al., 2012; Chen et al., 2014). To confirm FRRS1L-AMPAR association, we performed a co-immunoprecipitation (Co-IP) assay in human embryonic kidney 293T (HEK) cells. We found that HA-tagged FRRS1L (HA-FRRS1L) could be co-immunoprecipitated with Flag-tagged GluA1 (Flag-GluA1) or Flag-tagged GluA2 (FlagGluA2) subunit of the AMPAR from cells co-transfected with both constructs, but not from control cells transfected with either one (Figure 1A), indicating that FRRS1L can associate with both GluA1 and GluA2 in HEK cells. In addition, we found that in heterologous cells, FRRS1L did not form dimers or oligomers, as Myc-tagged FRRS1L (FRRS1L-Myc) was not coimmunoprecipitated by HA-FRRS1L in HEK cells (Figure 1B).

In mouse, FRRS1L protein was detected in the brain as well as in a few other tissues (Figure 1C). In the brain, FRRS1L had a broad distribution with high expression observed in hippocampus, cortex and thalamus (Figure 1D). In addition, expression of FRRS1L in the brain steadily increased during the development with high expression levels reached at $\sim$ postnatal two- to three-week-old (Figure 1E). In dissociated hippocampal primary neuron cultures, the majority of neuronal surface HAFRRS1L (sHA-FRRS1L) did not co-localize with an excitatory synapse marker, PSD-95 (Figure 1F), suggesting that FRRS1L may mainly exert its function outside of synapses. In addition, a substantial fraction of sHA-FRRS1L co-localized with a portion of endogenous surface GluA1 (sGluA1) (Figure 1F), indicating that FRRS1L associates with a subpopulation of GluA1-containing AMPARs in hippocampal neurons.

\section{FRRS1L Is Localized at Dynein-Positive Vesicles}

Microtubule-based transport by dynein and kinesin motors has been shown to play an important role in the regulation of AMPAR trafficking (Kim and Lisman, 2001; Setou et al., 2002; Kapitein et al., 2010; Hoerndli et al., 2013, 2015; Heisler et al., 2014). We were wondering whether FRRS1L shows any specificity to dynein or kinesin vesicles. To this end, we performed immunoisolation of vesicles in floated membrane fractions isolated from detergentfree hippocampal homogenate with sucrose step-gradients and utilized Western blotting to analyze the proteins associated biochemically with the vesicles (Figure 2A). We found that AMPARs, FRRS1L, TARP $\gamma 8$ and CNIH2 were detected in the PNSM, but not in the 35/40 fraction, suggesting that AMPARs and auxiliary subunits are not associated with the specific 35/40 membrane fraction (Figure 2B). Interestingly, in the vesicleenriched 8/35 fraction (Encalada et al., 2011), while AMPARs, FRRS1L, and TARP $\gamma 8$ were detected, CNIH2 was not detectable, indicating a diverse distribution of AMPAR auxiliary subunits in distinct membranous entities (Figure 2B).

In the $8 / 35$ membrane fraction, an antibody against dynein intermediate chain (DIC) pulled down dynein, but not one of the major kinesin heavy chains in neurons, KIF5B (Figure 2C). Significantly, we found AMPARs and FRRS1L, but not TARP $\gamma 8$ and $\mathrm{CNIH} 2$, in dynein vesicles, confirming an association of endogenous FRRS1L with dynein vesicles (Figure 2C). Interestingly, an antibody against KIF5B pulled down KIF5B and AMPARs, but none of three auxiliary subunits of AMPARs: FRRS1L, TARP $\gamma 8$, and CNIH2 (Figure 2D), suggesting that AMPARs in KIF5B vesicles unlikely contain FRRS1L, TARP $\gamma 8$ or CNIH2. However, we could not exclude the possibility that the levels of these auxiliary subunits in KIF5B immunoprecipitates in the $8 / 35$ membrane fraction may be below the detection thresholds of corresponding antibodies. Taken together, these biochemical data demonstrate that a portion of native FRRS1L associates with dynein vesicles. These data also indicate the existence of distinct subpopulations of hippocampal AMPARs that are decorated with unique combinations of auxiliary subunits.

\section{FRRS1L Does Not Change the Expression of AMPARs at the Plasma Membrane in HEK Cells}

We then sought to determine the role of FRRS1L in the regulation of AMPARs. Membrane proteins that interact with AMPARs often regulate AMPAR trafficking (Jackson and Nicoll, 2011; 

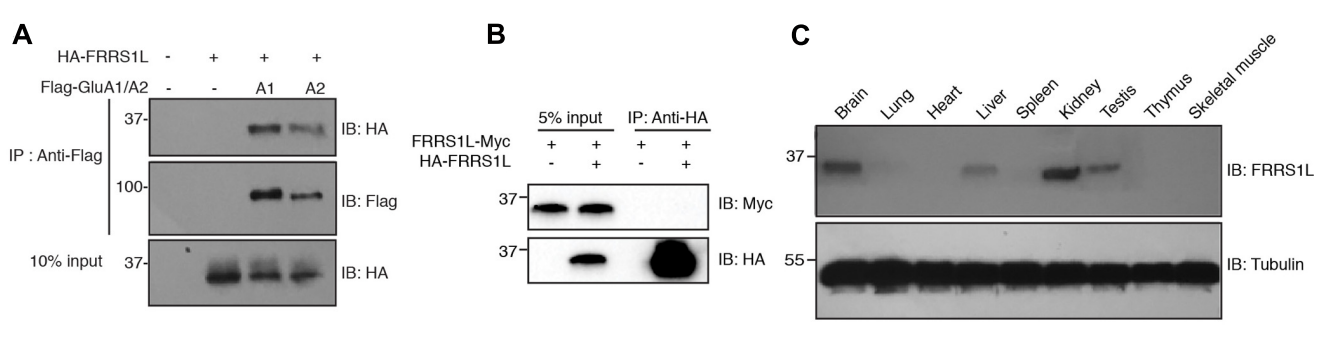

D

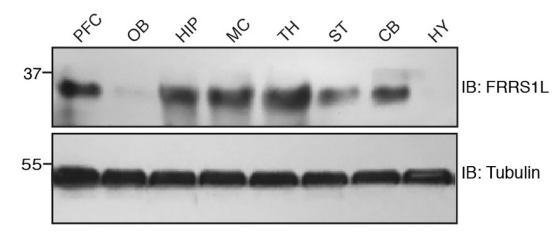

$\mathbf{F}$

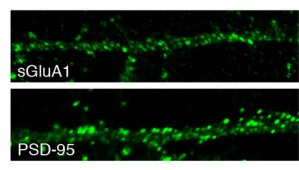

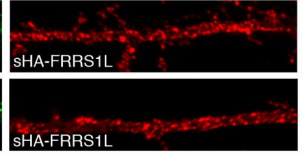

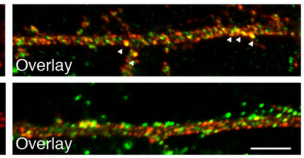

E
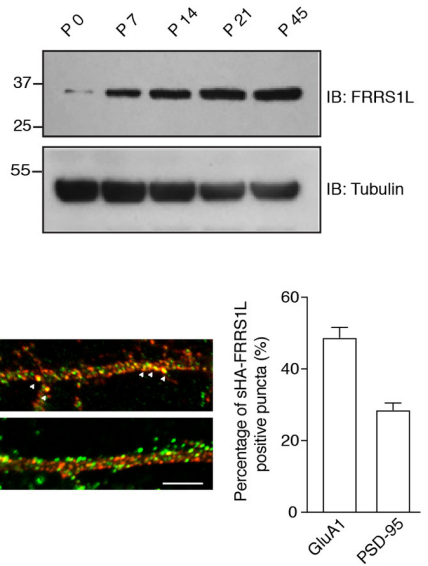

FIGURE 1 | Basic characterization of FRRS1L protein in mice. (A) Co-IP of FRRS1L with GluA1 or GluA2(Q) in HEK cells. Lysates from HEK cells transfected with HA-FRRS1L alone, HA-FRRS1L together with Flag- GluA1 or GluA2(Q), or an empty vector as a control, were immunoprecipitated with an anti-Flag antibody. The IPs and $10 \%$ input were probed with indicated antibodies. IB, immunoblotting. $N=3$ independent repeats. (B) FRRS1L did not form dimers or oligomers in HEK cells. Lysates from HEK cells transfected with FRRS1L-Myc alone or together with HA-FRRS1L were immunoprecipitated with an anti-HA antibody. The IPs and 5\% input were probed with indicated antibodies. $N=3$ independent repeats. (C) Tissue distribution of endogenous FRRS1L in adult mouse. $N=4, n=5$ animals. (D) Expression profile of FRRS1L in adult mouse brain tissues. PFC, prefrontal cortex; OB, olfactory bulb; HIP, hippocampus; MC, motor cortex; TH, thalamus; ST, striatum; CB, cerebellum; HY, hypothalamus. $N=3$ independent repeats from 6 animals. (E) Developmental profile of FRRS1L expression in mouse hippocampus. $N=4$ independent repeats. (F) Representative images show that surface HA-FRRS1L (sHA-FRRS1L) in mouse dissociated hippocampal neuron cultures at DIV16 partially co-localizes with surface GluA1 (sGluA1), but the majority of sHA-FRRS1L does not co-localize with an excitatory synapse marker, PSD-95. Arrowheads indicate the co-localization of SHA-FRRS1L with sGluA1. The bar graph in right shows the percentage of co-localization. Scale bar, $20 \mu \mathrm{m}$. $N=3$ independent repeats. Uncropped scans of Western blots in Supplementary Figures 1A-E.

Straub and Tomita, 2012). To study FRRS1L-mediated effect on AMPAR trafficking, we first expressed AMPAR subunits on their own or together with FRRS1L in HEK cells and employed an immunocytochemical assay to examine the ratio of surface to total expression levels of AMPAR subunits. In HEK cells, HA-FRRS1L on its own could traffic to the cell surface (Figure 3A). In addition, sHA-FRRS1L co-localized with surface Flag-GluA1 (sFlag-GluA1) or surface Flag-GluA2 (Q) (sFlagGluA2) in HEK cells (Figure 3B), indicating that FRRS1L is associated with AMPARs on the cell surface. Furthermore, we found that levels of sFlag-GluA1 or sFlag-GluA2 in HEK cells were not altered by co-expression with FRRS1L-IRES-mCherry (Figures 3C,D). We also co-expressed FRRS1L-IRES-mCherry together with GluA1-IRES-EGFP or GluA2-IRES-EGFP in HEK cells and conducted patch clamp recordings to measure AMPARmediated whole-cell currents. We found that the AMPAR subunit expressed on its own generated the same amount of wholecell currents evoked by $1 \mathrm{mM}$ glutamate (in the presence of cyclothiazide to block receptor desensitization) as the AMPAR subunit and FRRS1L expressed together (Figures 3E,F). Taken together, these data indicate that the abundance of surface
AMPAR subunits is not changed by FRRS1L co-expression in HEK cells.

\section{Over-Expression of FRRS1L Does Not Affect AMPAR-Mediated Synaptic Transmission in Hippocampal CA1 Neurons}

To study the role of FRRS1L in neurons, we first performed an immunocytochemical assay to measure surface and total levels of GluA1, a major AMPAR subunit expressed in hippocampal neurons (Lu et al., 2009), in dissociated primary hippocampal cultures that were transfected with FRRS1L-IRES-EGFP. Double immunolabeling of surface and total GluA1 demonstrated that over-expression of FRRS1L did not change surface levels, total levels, or surface to total ratio of GluA1 expression (Figure 4A), suggesting that increased expression of FRRS1L is not sufficient to alter AMPAR trafficking to the neuronal surface.

To corroborate the immunocytochemical data, we performed electrophysiological recordings to measure AMPAR-mediated 

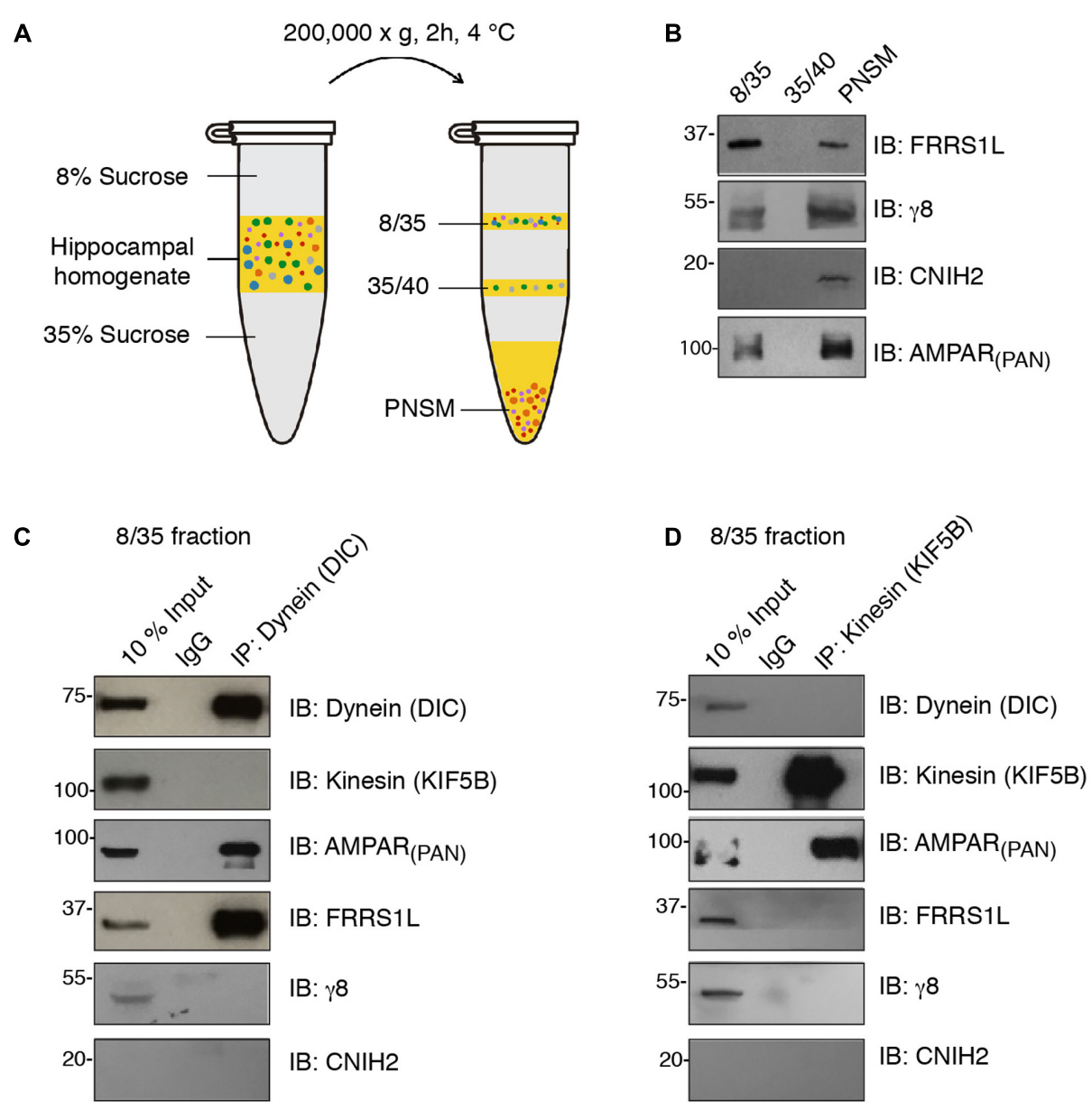

FIGURE 2 | FRRS1L is localized at dynein, but not kinesin, vesicles in hippocampus. (A) The schematic of vesicle membrane preparation from detergent-free hippocampal homogenate. (B) Differential distributions of FRRS1L, $\gamma 8, \mathrm{CNIH}$, and the AMPAR (probed by a pan-AMPAR antibody) in 8/35, 35/40, and post-ultracentrifugation PNS membrane fractions (PNSM) ( $N=4$ independent repeats). (C,D) Vesicle immunoisolation of $8 / 35$ membrane fraction by a dynein (C, the antibody was against the dynein intermediate chain, DIC) or kinesin (D, the antibody was against the KIF5B) antibodies. The 10\% input was also included. The immunoprecipitates were mixed with SDS-PAGE sample buffer and subjected to Western blotting by indicated antibodies ( $N=5$ independent repeats). Uncropped scans of Western blots in Supplementary Figures 2B-D.

synaptic transmission in neurons over-expressing FRRS1L. Toward this end, we biolistically transfected cultured organotypic hippocampal slices with gold particles that were coated with plasmids expressing FRRS1L-IRES-EGFP. Two to five days after transfection, we performed simultaneous dual whole-cell voltage clamp recordings (dual recordings) that were made from a transfected pyramidal cell (identified by EGFP) and a neighboring EGFP-negative neuron to detect AMPAR- and NMDA receptor (NMDAR)-mediated EPSCs. A stimulating electrode was placed at the Schaffer collateral pathway to evoke EPSCs in both cells. We found that over-expression of FRRS1L did not change either AMPA or NMDA EPSCs (Figures 4B,C), and the weighted decay time constant of the AMPA EPSC (Figure 4D). In addition, the PPR, a measure of presynaptic neurotransmitter release probability, of AMPA EPSCs in the postsynaptic neurons over-expressing FRRS1L was not changed (Figure 4E). These data indicate that over-expression of FRRS1L in hippocampal pyramidal neurons does not affect the strength of excitatory synaptic transmission.

\section{Genetic Deletion of FRRS1L Significantly Reduces Surface AMPARs in Neurons}

To further determine the physiological role of FRRS1L protein in neurons, we utilized the CRISPR-Cas9 system to develop single-guide RNAs (sgRNAs) to target Frrs1l gene loci in mouse genome. Among three FRRS1L sgRNA candidates, sgRNA candidate $1\left(\operatorname{sgRNA}^{\# 1}\right)$ did not decrease the expression of co-transfected FRRS1L-Myc in HEK cells, while the other two candidates (sgRNA ${ }^{\# 2}$ and $\operatorname{sgRNA}^{\# 3}$ ) effectively reduced the expression levels of FRRS1L-Myc (Figure 5A). We further tested the effectiveness of sgRNA and sgRNA ${ }^{\# 3}$ in inactivating the Frrsll gene in neurons (sgRNA vectors simultaneously expressed EGFP for cell identification) with an in situ hybridization assay to measure FRRS1L mRNAs in dissociated hippocampal primary cultures 
A
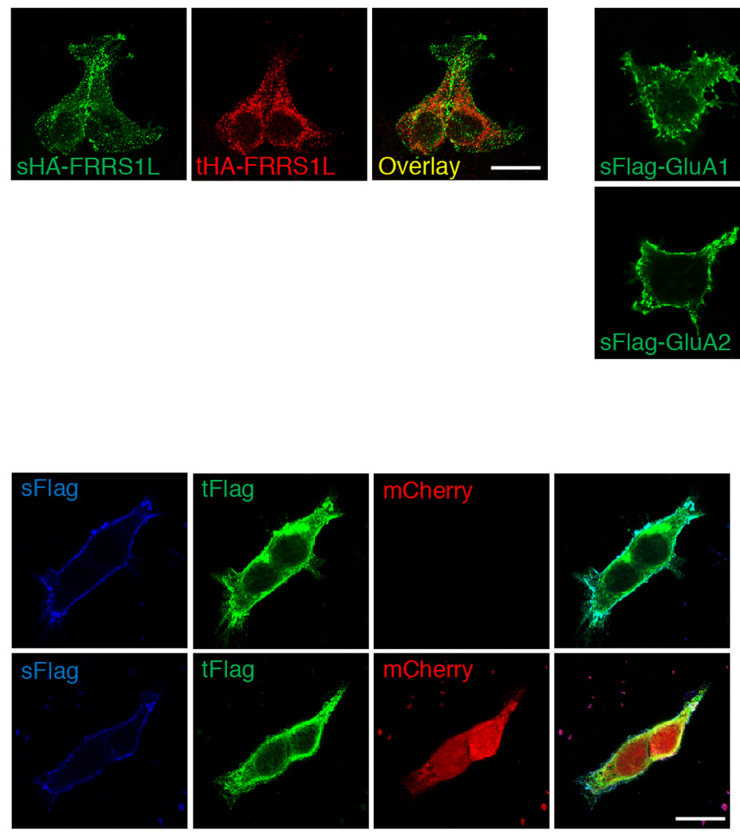

D
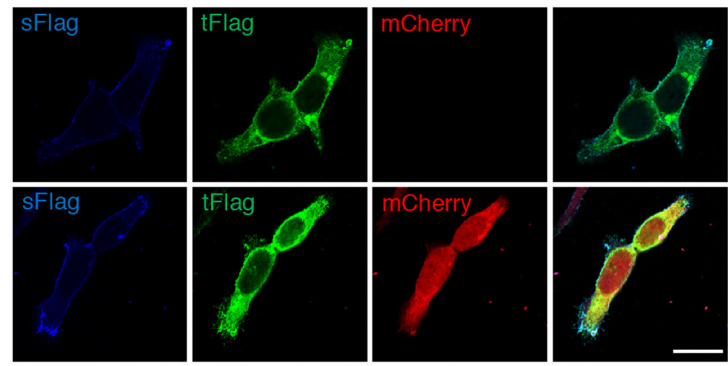

B
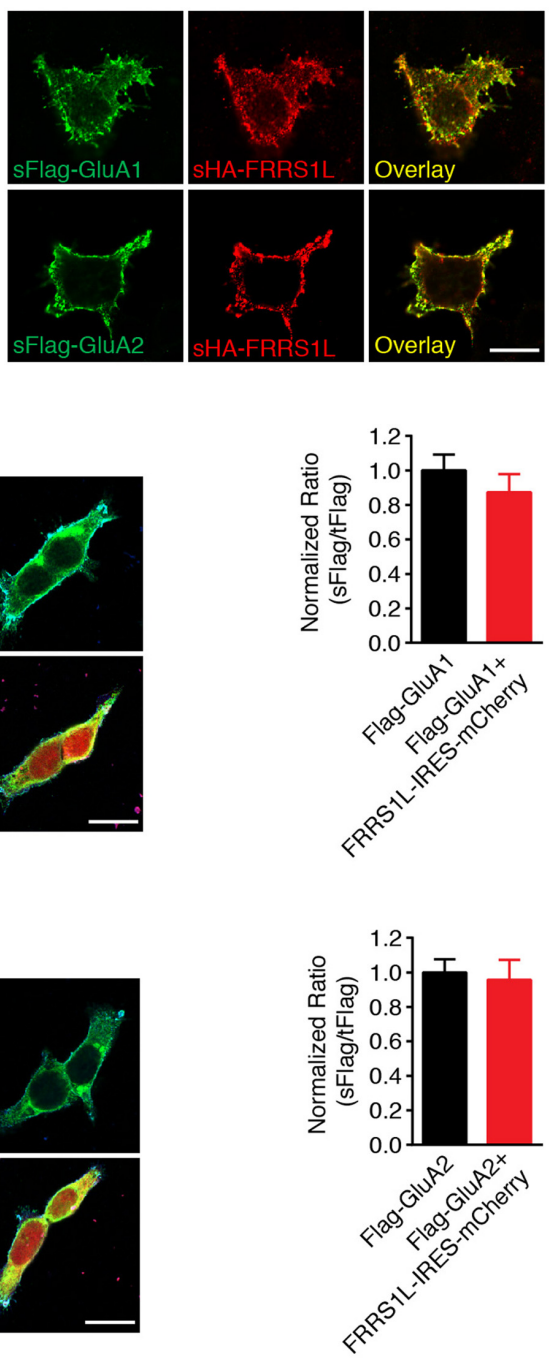

E

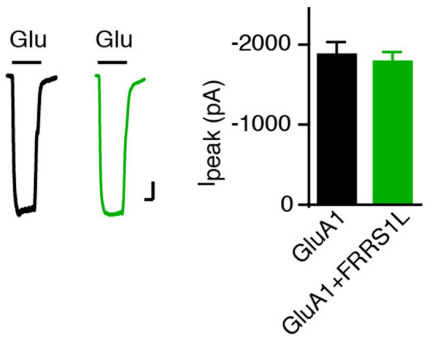

F

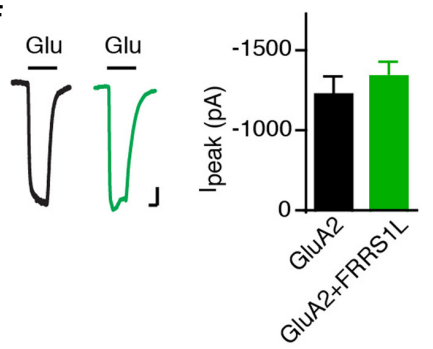

FIGURE 3 |FRRS1L does not change the expression of AMPARs at the plasma membrane in HEK cells. (A) Representative images of surface and total HA-FRRS1L show that FRRS1L was expressed at the HEK cell surface. Scale bar, $20 \mu \mathrm{m}$. (B) Representative images of sHA-FRRS1L and sFlag-GluA1 (top) or sFlag-GluA2(Q) (bottom) show that sHA-FRRS1L co-localized with sFlag-GluA1 or sFlag-GluA2. Scale bar, $20 \mu \mathrm{m}$. (C,D) Representative images of HEK cells in left panels show that FRRS1L did not change the ratio of surface and total AMPAR expression. HEK cells were transfected with Flag-GluA1 (C) or Flag-GluA2(Q) (D) on its own, or together with FRRS1L-IRES-mCherry. Bar graphs at right show normalized ratio of surface to total GluA1 (Flag-GluA1: $n=15$; Flag-GluA1 + FRRS1L-IRESmCherry: $n=21, p=0.40$, $t$-test) or GluA2 (Flag-GluA2: $n=15$; Flag-GluA2 + FRRS1L-IRES-mCherry: $n=20, p=0.77, t$-test) expression. sFlag, surface Flag; tFlag, total Flag. Scale bar, $20 \mu \mathrm{m}$. (E,F) FRRS1L did not affect GluA1- (E, GluA1: $n=18$; GluA1 + FRRS1L: $n=25, p=0.65, t$-test) or GluA2(Q)- (F, GluA2: $n=17$; GluA2 + FRRS1L: $n=25, p=0.82$, $t$-test) mediated, 1 mM glutamate-evoked whole-cell currents in the presence of cyclothiazide in HEK cells. Glu, glutamate. Scale bar, 200 pA and 500 ms. 

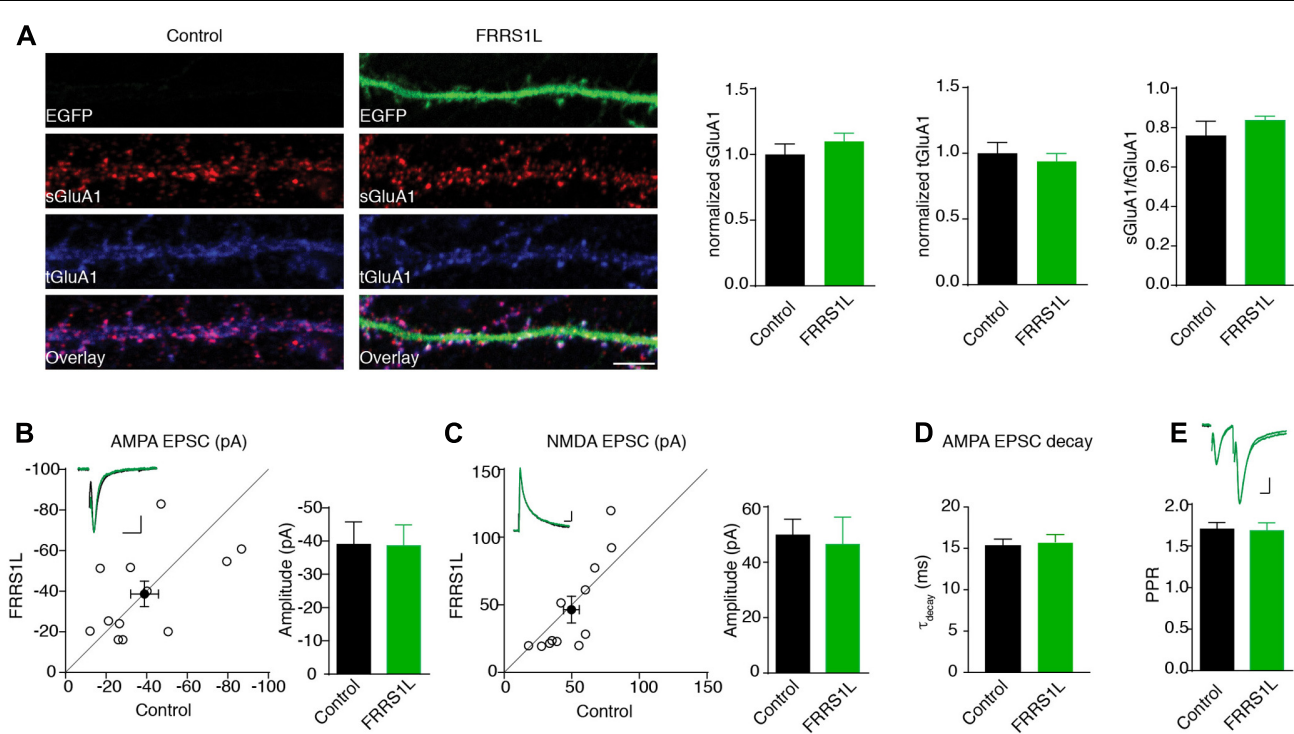

D
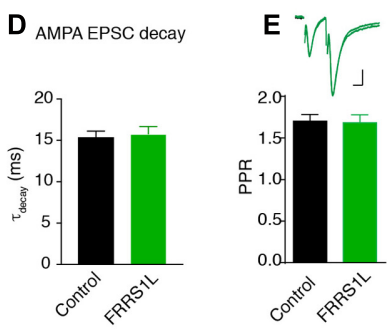

FIGURE 4 | Over-expression of FRRS1L does not change AMPA receptor-mediated synaptic transmission. (A) Representative image of dendrites shows that over-expression of FRRS1L did not change GluA1 surface and total levels nor the ratio of GluA1 surface to total expression in cultured hippocampal neurons (Control: $n=12$; FRRS1L: $n=26$, sGluA1: $p=0.36$; tGluA1: $p=0.57$; sGluA1/tGluA1: $p=0.21$, $t$-test). (B,C) Over-expression of FRRS1L did not change AMPA (B, $n=12, p=0.96$, paired $t$-test) and NMDA $(\mathbf{C}, n=12, p=0.10$, paired $t$-test) EPSCs of hippocampal pyramidal neurons in organotypic hippocampal slice cultures. The open circles represent all pair recordings, the solid circle shows the average of all pair recordings. Scale bar, 20 pA and 100 ms for AMPA EPSC; 20 pA and $40 \mathrm{~ms}$ for NMDA EPSC. (D) The weighted decay time constant of AMPA EPSCs was not changed in neurons over-expressing FRRS1L ( $n=12$ for both conditions, $p=0.46$, $t$-test). (E) The paired pulse ratio (PPR) was not changed in neurons over-expressing FRRS1L ( $n=12, p=0.81, t$-test). Scale bar, $20 \mathrm{pA}$ and $100 \mathrm{~ms}$.

A

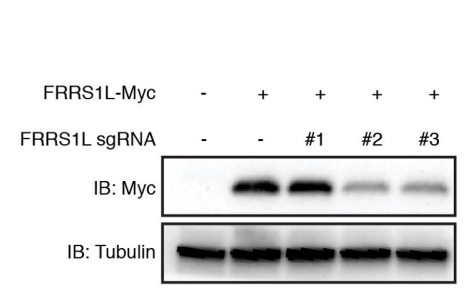

B

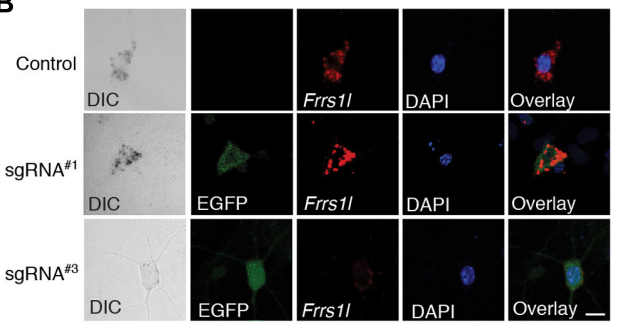

C

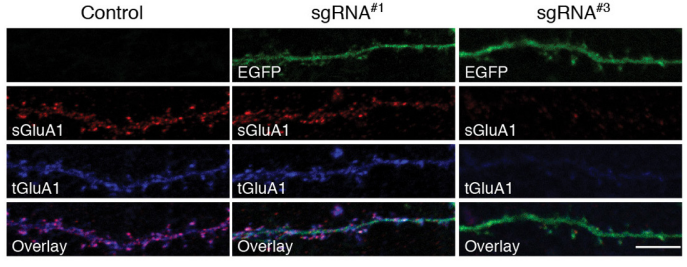

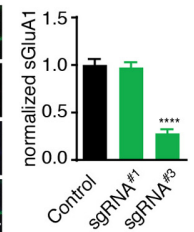

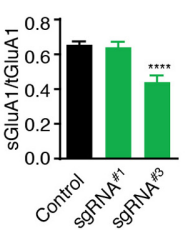

FIGURE 5 | Genetic deletion of FRRS1L reduces the surface and total expression levels of GluA1 in cultured hippocampal neurons. (A) Screening candidate sgRNAs against FRRS1L in HEK cells. Western blotting analysis show that sgRNA ${ }^{\# 2}$ and sgRNA ${ }^{\# 3}$, but not sgRNA ${ }^{\# 1}$, strongly reduced FRRS1L-Myc expression in HEK cells, $N=3$ independent repeats. Uncropped scans of Western blots were shown in Supplementary Figure 3. (B) In situ hybridization (ISH) data show that sgRNA ${ }^{\# 3}$, but not sgRNA ${ }^{\# 1}$, eliminated Frrs 1/ mRNA expression (in red) in cultured hippocampal neurons (Control: score 3, $n=20$; sgRNA $\# 1$ : score 3, $n=15$; sgRNA ${ }^{\# 3}$ : score $\left.0-1, n=18\right)$. Scale bar, $10 \mu \mathrm{m}$. (C) sgRNA $\# 3$ induced genetic deletion of FRRS1L impaired sGluA1 and tGluA1 expressions in cultured hippocampal neurons. Representative dendrite images from a non-transfected control neuron or neurons expressing FRRS1L sgRNAs (Control: $n=11$; sgRNA ${ }^{\# 1}$ : $n=14$; sgRNA $\# 3: n=13$; normalized sGluA1: $F(2,38)=12.58, p<0.0001$; normalized tGluA1: $F(2,38)=16.74, p<0.0001$, sGluA1/tGluA1: F $(2,38)=9.27$, $p<0.0001$; one-way ANOVA with post hoc Fisher's LSD test) are shown in the left panels. Scale bar, $20 \mu \mathrm{m}$. 
(Commercially available FRRS1L antibodies were not suitable for immunolabeling of endogenous FRRS1L in neurons, and thus we could not perform immunocytochemical experiments in neurons). We found that in neurons expressing $\operatorname{sgRNA}^{\# 3}$, FRRS1L mRNAs were essentially not detectable as compared to FRRS1L mRNAs in non-transfected neurons (Control) or sgRNA $^{\# 1}$-expressing neurons (Figure 5B), indicating that we had successfully achieved single-cell $\mathrm{KO}$ of FRRS1L in neurons expressing sgRNA ${ }^{\# 3}$.

To study the role of FRRS1L in AMPAR trafficking to the plasma membrane in neurons, we transfected sgRNA ${ }^{\# 3}$, or sgRNA ${ }^{\# 1}$ as a control, at DIV2 (days in vitro) neuronal cultures and measured surface and total GluA1 expression levels at DIV16. We found that surface levels of GluA1 were significantly reduced in neurons expressing sgRNA ${ }^{\# 3}$ (Figure 5C). Interestingly, total GluA1 expression levels were also reduced in sgRNA ${ }^{\# 3}$-expressing neurons (Figure 5C), suggesting that FRRS1L is critical for the stability of GluA1 protein in neurons. Furthermore, the surface to total ratio of GluA1 expression was significantly decreased in neurons expressing sgRNA $^{\# 3}$ (Figure 5C), indicating that targeting of GluA1 to the neuronal surface is impaired in neurons lacking FRRS1L. In contrast, transfection of sgRNA ${ }^{\# 1}$ into neurons changed neither surface nor total GluA1 (Figure 5C). Taken together, these data demonstrate that FRRS1L is critical for GluA1 stability in neurons and also plays a role in GluA1 trafficking to the neuronal surface.

\section{FRRS1L Is Important for AMPAR-Mediated Synaptic Transmission in Vitro and in Vivo}

Finally, we examined the effect of single-cell KO of FRRS1L on AMPAR-mediated synaptic transmission. We first biolistically transfected cultured organotypic hippocampal slices with sgRNA $^{\# 3}$ or sgRNA $^{\# 1}$ plasmids. Approximately 2 weeks after transfection, we performed dual recordings to measure glutamatergic synaptic transmission in pyramidal neurons. Expression of $\operatorname{sgRNA}^{\# 3}$ specifically reduced the amplitude of AMPA EPSCs by $\sim 30 \%$, without affecting NMDA EPSC amplitudes (Figure 6A). In addition, there was no change of PPR (Figure 6C), suggesting that loss of FRRS1L in the postsynaptic neurons does not alter presynaptic neurotransmitter release probability. In contrast, expression of sgRNA ${ }^{\# 1}$ did not affect AMPA and NMDA EPSCs nor PPR (Figures 6D,F). Importantly, an sgRNA ${ }^{\# 3}$-resistant FRRS1L mutant (FRRS1L*) fully rescued the deficit of AMPA EPSCs in pyramidal neurons expressing sgRNA $^{\# 3}$ (Figures 6G,H), demonstrating that the effect of sgRNA $^{\# 3}$ on AMPA EPSCs is due to the loss of FRRS1L protein. Furthermore, none of the manipulations changed the weighted decay time constants of AMPA EPSCs (Figures 6B,E,I). Taken together, these results show that FRRS1L is critical for AMPARmediated synaptic transmission in hippocampal pyramidal neurons.

To study the role of FRRS1L in the regulation of glutamatergic synaptic transmission in vivo, we electroporated plasmids in utero to sparsely express sgRNA ${ }^{\# 3}$ in hippocampal progenitor cells in E14.5 mice embryos to inactivate Frrs $1 l$ alleles and then performed dual recordings to measure glutamatergic synaptic transmission in acute hippocampal slices prepared from 2- to 3-week-old mice. We found that single-cell KO of FRRS1L with sgRNA ${ }^{\# 3}$ in CA1 pyramidal neurons in acute hippocampal slices significantly decreased AMPA, but not NMDA, EPSCs (Figure 6J). In addition, there was no change of the weighted decay time constant of AMPA EPSCs and PPR (Figures 6K,L). Thus, FRRS1L is important for AMPAR-mediated synaptic transmission in vivo.

\section{DISCUSSION}

Recent proteomic screenings have identified a growing number of membrane proteins that are associated with native AMPAR complexes in the brain (Schwenk et al., 2009, 2012; von Engelhardt et al., 2010; Chen et al., 2014; Bettler and Fakler, 2017). Subsequent functional investigations have revealed that many of these membrane proteins play critical roles in the regulation of trafficking and/or function of AMPARs (Schwenk et al., 2009, 2012; Kato et al., 2010; Shi et al., 2010; von Engelhardt et al., 2010; Gill et al., 2011, 2012; Coombs et al., 2012; Herring et al., 2013; Boudkkazi et al., 2014; Gratacos-Batlle et al., 2014; Fado et al., 2015; McGee et al., 2015; Erlenhardt et al., 2016; Gu et al., 2016a; Klaassen et al., 2016; Wei et al., 2016; Mao et al., 2017). In this study, we have focused on FRRS1L, a protein isolated in AMPAR complexes in the brain, but its function in the regulation of AMPAR-mediated synaptic transmission was unclear. Underscoring the importance of FRRS1L in neuronal function, recent human genetic studies have shown that lossof-function mutations of FRRS1L in human lead to severe impairments of motor and cognitive functions (Madeo et al., 2016; Shaheen et al., 2016).

Immunocytochemical data in neuronal cultures show that only a minor fraction of immunolabeling of expressed FRRS1L co-localizes with the excitatory synaptic marker, PSD-95, indicating that FRRS1L is largely localized at non-synaptic subcellular regions in neuronal dendrites. Moreover, there is a substantial co-localization between expressed FRRS1L and endogenous AMPAR subunit GluA1, suggesting that FRRS1L associates with a subpopulation of AMPARs. Interestingly, our data demonstrate that FRRS1L, but not TARP $\gamma 8$ or CNIH2, associates with dynein, but not kinesin KIF5B, vesicles. Previous studies have shown that microtubule-based motors, kinesin and dynein, are important for the long-range dendritic transport of vesicles containing the assembled AMPARs out of the ER and other compartments of the endomembrane system, and for the regulation of excitatory synaptic transmission (Kim and Lisman, 2001; Setou et al., 2002; Kapitein et al., 2010; Hoerndli et al., 2013). The association of FRRS1L with dynein vesicles suggests that FRRS1L-containing AMPARs could be transported by dynein-dependent mechanisms. However, the exact role of FRRS1L in dynein-based AMPAR trafficking remains to be examined.

In heterologous HEK cells, co-expression of FRRS1L with the AMPAR subunit GluA1 or GluA2 does not change the surface abundance of the receptor subunits. Similarly, expression 


\section{A}

FRRS1L sgRNA*3

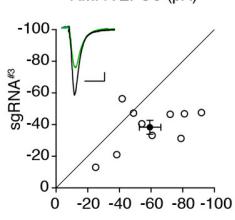

Control

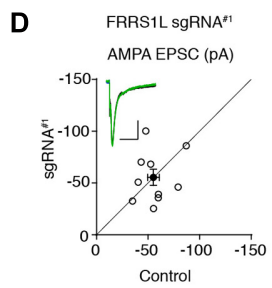

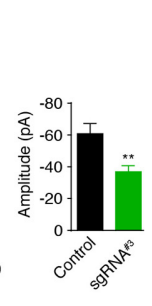

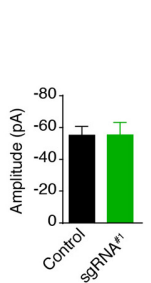

FRRS1L sgRNA ${ }^{* 3}$

$\operatorname{NMDA} \operatorname{EPSC}(\mathrm{pA})$

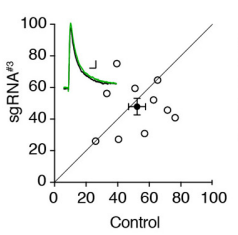

Control

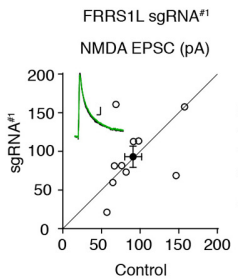

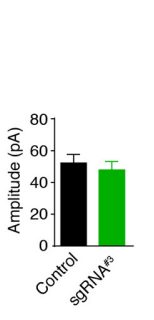

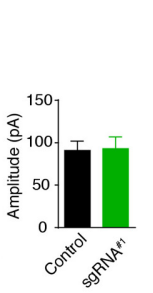

B
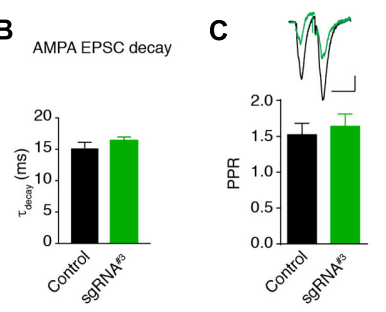

E AMPA EPSC decay

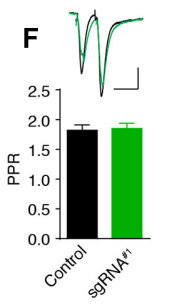

G

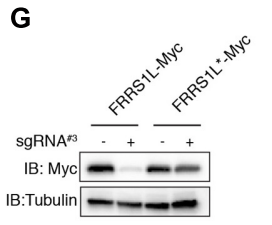

H sgRNA $^{* 3}+$ FRRS1L $^{*}$ (Rescue) AMPA EPSC (PA)

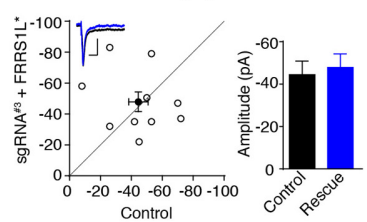

Control

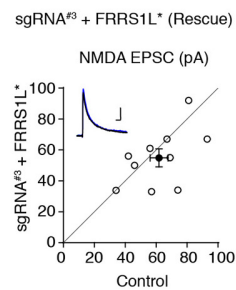

Control

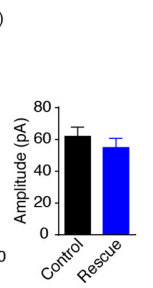

K

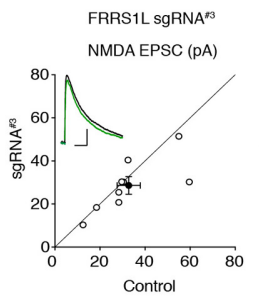

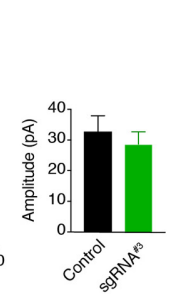

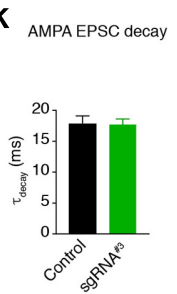

I AMPA EPSC decay

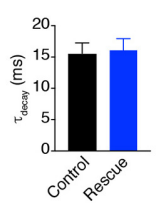

FIGURE 6 | Single-cell genetic deletion of FRRS1L in vitro and in vivo significantly reduces AMPAR-mediated synaptic transmission. (A) Scatter plot of AMPA and NMDA EPSCs in pyramidal neurons expressing sgRNA $\# 3$ and nearby control neurons in cultured organotypic hippocampal slices. Expression of sgRNA ${ }^{\# 3}$ significantly reduced AMPA EPSCs ( $n=10, p<0.01$, paired $t$-test), without affecting NMDA EPSCs ( $n=10, p=0.56$, paired $t$-test). Scale bar, 20 pA and 100 ms. (B) The weighted decay time constant was not changed in neurons expressing sgRNA $\# 3$ ( $n=10, p=0.25$, $t$-test). (C) There was no change of PPR in neurons expressing sgRNA ${ }^{\# 3}(n=11, p=0.36$, $t$-test). Scale bar, 20 pA and 100 ms. (D) Scatter plot of AMPA and NMDA EPSCs in pyramidal neurons expressing sgRNA ${ }^{\# 1}$ and nearby control neurons in cultured organotypic hippocampal slices. Expression of sgRNA $\# 1$ did not change AMPA ( $n=10, p=0.98$, paired $t$-test) and NMDA ( $n=10, p=0.88$, paired $t$-test) EPSCs. Each open circle represents one paired recording in the scatter plots, and the solid black point is the average of all pair recordings. Scale bar, $20 \mathrm{pA}$ and $100 \mathrm{~ms}$. (E) The weighted decay time constant was not changed in neurons expressing sgRNA ${ }^{\# 1}$ ( $n=10, p=0.97, t$-test). (F) There was no change of PPR in neurons expressing sgRNA ${ }^{\# 1}$ ( $n=10, p=0.76$, $t$-test). Scale bar, $20 \mathrm{pA}$ and $100 \mathrm{~ms}$. (G) sgRNA $\# 3$ failed to reduce the expression of sgRNA resistant FRRS1L (FRRS1L*) in HEK cells. Western blotting analysis showed that sgRNA ${ }^{\# 3}$ strongly reduced WT FRRS1L-Myc, but not FRRS1L-Myc*, expression in HEK cells. Uncropped scans of Western blots were shown in Supplementary Figure 3. (H) Scatter plot of AMPA and NMDA EPSCs in pyramidal neurons expressing sgRNA ${ }^{\# 3}$ together with FRRS1L* and nearby control neurons in cultured organotypic hippocampal slices. Expression of FRRS1L* rescued the AMPA EPSC deficit induced by sgRNA $\# 3$ (AMPA, $n=10, p=0.74$; NMDA, $n=10, p=0.24$; paired $t$-test). Scale bar, 20 pA and 100 ms. (I) The weighted decay time constant was not changed in neurons expressing sgRNA ${ }^{\# 3}$ together with FRRS1L* ( $n=10, p=0.85, t$-test). ( $\left.\mathbf{J}\right)$ Scatter plot of AMPA and NMDA EPSCs in CA1 neurons expressing sgRNA ${ }^{\# 3}$ and nearby control neurons in acute hippocampal slices from p14-16 mice that were electroporated in utero at E14.5-15.5. Expression of sgRNA $\# 3$ in vivo significantly reduced AMPA EPSCs ( $p<0.05, n=9$, paired $t$-test with Wilcoxon test), without affecting NMDA EPSCs ( $n=9, p=0.53$, paired $t$-test). Scale bar, 20 pA and 100 ms. (K) The weighted decay time constant was not changed in neurons expressing sgRNA ${ }^{\# 3}$ ( $n=9$, $p=0.88, t$-test). (L) sgRNA ${ }^{\# 3}$ did not change the PPR (control and sgRNA $\# 3: n=9, p=0.81, t$-test). Scale bar, 20 pA and 100 ms.

of AMPAR subunits together with FRRS1L does not alter $1 \mathrm{mM}$ glutamate-evoked whole-cell currents in the presence of cyclothiazide in HEK cells. Thus, FRRS1L on its own appears not be sufficient to change the expression of AMPARs at the plasma membrane, which is different from other AMPAR auxiliary subunits that often either promote or diminish AMPARs at the cell surface in heterologous cells (Tomita et al., 2004; Schwenk et al., 2009; von Engelhardt et al., 2010; 
Shanks et al., 2012; Gratacos-Batlle et al., 2014; Gu et al., 2016a; Erlenhardt et al., 2016; Wei et al., 2016). Furthermore, in neurons, over-expression of FRRS1L changes neither the surface GluA1 nor AMPAR-mediated synaptic transmission. These data indicate that increased expression of FRRS1L in neurons is not sufficient to alter AMPAR trafficking to the plasma membrane and FRRS1L is unlikely a limiting factor in determining the expression levels of AMPARs at the surface of cultured neurons in our experiments. Our findings that over-expression of FRRS1L does not change excitatory synaptic transmission and that single-cell genetic deletion of FRRS1L decreases AMPAR-mediated EPSCs resemble previous work on CNIH2. In hippocampal CA1 neurons, it has been reported that over-expression of $\mathrm{CNIH} 2$ in hippocampal neurons does not change excitatory synaptic transmission (Herring et al., 2013). However, genetic deletion of CNIH2 in hippocampal CA1 neurons strongly diminishes AMPAR-mediated synaptic transmission (Herring et al., 2013). In addition, a recent study has shown that in HEK cells, FRRS1L (C9orf4) can reduce non-saturating $100 \mu \mathrm{M}$ glutamate-evoked, GluA1 homomermediated calcium influx, but increase GluA1-TARP $\gamma 8$ complexmediated calcium influx (Erlenhardt et al., 2016). These data suggest that while FRRS1L does not change the number of receptors at the cell surface as shown in our study, it can modulate the steady-state currents in the absence of cyclothiazide and can functionally modulate AMPAR-TARP $\gamma 8$ complexes in heterologous cells.

sgRNA-based single-cell KO of FRRS1L shows that FRRS1L plays an important role in the regulation of AMPAR-mediated synaptic transmission in hippocampal pyramidal neurons. Indeed, FRRS1L $\mathrm{KO}$ in hippocampal neurons both in vitro and in vivo reduces the AMPA EPSCs. Importantly, a sgRNA resistant FRRS1L fully rescues the AMPA EPSC deficit in the sgRNA-positive neurons. Similarly, FRRS1L KO in neuronal cultures significantly reduces GluA1 staining on the neuronal surface. These data are in agreement with a previous study in a neuronally differentiated cell line in which knockdown of FRRS1L diminished AMPA-induced whole-cell currents (Madeo et al., 2016). In addition, GluA1 total protein levels are reduced in FRRS1L KO neurons, suggesting that FRRS1L is important for GluA1 stability and/or biogenesis. Furthermore, the surface to total ratio of GluA1 expression is significantly decreased in neurons lacking FRRS1L. Thus, it is likely that decreased surface levels of GluA1 in FRRS1L KO neurons are caused by a combined effect of reduced total GluA1 expression and impaired delivery of GluA1 to the neuronal surface. It has been reported that expression levels of AMPAR receptor subunits are reduced in neurons lacking other AMPAR auxiliary subunits, including TARPs, CNIHs, PORCN or CKAMP44 (Rouach et al., 2005; Herring et al., 2013; Khodosevich et al., 2014; Erlenhardt et al., 2016), indicating that AMPAR auxiliary subunits commonly play important roles in the regulation of AMPAR stability in neurons.

While this manuscript was in preparation, an elegant study on FRRS1L that reveals a critical role of FRRS1L in early biogenesis of AMPARs and brain function was published (Brechet et al., 2017). This work shows that endogenous FRRS1L is largely confined in the ER, is important for AMPAR biogenesis and is required for AMPAR-mediated synaptic transmission (Brechet et al., 2017). Our data support these conclusions (Brechet et al., 2017). Indeed, similar to our single-cell KO data, it was reported that shRNA-mediated knockdown of FRRS1L diminished AMPAR-medicated synaptic transmission in hippocampal neurons (Brechet et al., 2017). In addition, our data show the reduced expression levels of GluA1 in FRRS1L KO neurons (Figure 5C), which is consistent with a role of FRRS1L in AMPAR biogenesis (Brechet et al., 2017). We also found that over-expression or single-cell $\mathrm{KO}$ of FRRS1L in hippocampal neurons did not change the AMPA EPSC decay kinetics, which is in agreement with the recent report (Brechet et al., 2017). Furthermore, our data show that FRRS1L, but not TARP $\gamma 8$ or CNIH2, associates with dynein, indicating that FRRS1L-containing AMPARs represent a distinct receptor population, consistent with the reported proteomic analysis of the unique FRRS1L-AMPAR assemblies (Brechet et al., 2017). It has also been reported that over-expression of FRRS1L increases AMPAR-mediated synaptic transmission (Brechet et al., 2017), which is different from our findings that FRRS1L over-expression did not change AMPA EPSCs. This discrepancy can be explained by different over-expression techniques (viral transduction in vivo versus plasmid transfection in vitro in our study), different experimental preparations (acute rat hippocampal slices versus mouse hippocampal organotypic cultures in our study), different neuronal types for recording (hippocampal mossy cells, interneurons or CA3 neurons versus hippocampal CA1 neurons in our study) and different overexpression durations ( $\sim 2$ weeks versus $2-5$ days in our study). It is possible that over-expression of FRRS1L in neurons may not represent the appropriate approach to study the FRRS1L function in synaptic transmission, as over-expressed FRRS1L can reach the neuronal surface (Figure 1F), but the vast majority of native FRRS1L do not (Brechet et al., 2017). Nevertheless, our findings of FRRS1L localization at dynein-positive vesicles together with the published report (Brechet et al., 2017) suggest that FRRS1L may primarily regulate the strength of AMPARmediated synaptic transmission through the modulation of AMPAR biogenesis and/or transport at the early secretory pathways.

In summary, we have provided some basic characterizations of FRRS1L in heterologous cells and hippocampal neurons. Our findings reveal an important role of FRRS1L in the regulation of excitatory synaptic transmission. Future work examining the molecular mechanisms underlying the regulation of AMPARs by FRRS1L and the potential role of dynein in the transport of FRRS1L-containing AMPARs will contribute to our understanding of AMPAR biogenesis and trafficking.

\section{AUTHOR CONTRIBUTIONS}

WH, HW, JL, SZ, and WL designed the research. HW performed immunocytochemical experiments in neurons, and electrophysiological recordings in HEK cells and hippocampal 
slice cultures. WH performed vesicle immunoisolation, immunocytochemical experiments in neuronal cultures and dual whole-cell current recording in acute hippocampal slices. WH and HW performed biochemical characterization. JL performed immunostaining in HEK cells. WL and WH wrote the paper. All authors read and commented on the manuscript, and approved the final version of the manuscript.

\section{FUNDING}

This work was supported by NINDS/NIH Intramural Research Program to WL, and by China National Natural Science Funds for Young Scholar (H0929-81701363) and China Scholarship Council to HW. The content is solely the responsibility of the

\section{REFERENCES}

Anggono, V., and Huganir, R. L. (2012). Regulation of AMPA receptor trafficking and synaptic plasticity. Curr. Opin. Neurobiol. 22, 461-469. doi: 10.1016/j.conb. 2011.12.006

Bettler, B., and Fakler, B. (2017). Ionotropic AMPA-type glutamate and metabotropic GABAB receptors: determining cellular physiology by proteomes. Curr. Opin. Neurobiol. 45, 16-23. doi: 10.1016/j.conb.2017.02.011

Boudkkazi, S., Brechet, A., Schwenk, J., and Fakler, B. (2014). Cornichon2 dictates the time course of excitatory transmission at individual hippocampal synapses. Neuron 82, 848-858. doi: 10.1016/j.neuron.2014.03.031

Brechet, A., Buchert, R., Schwenk J., Boudkkazi, S., Zolles, G., Siquier-Pernet, K., et al. (2017). AMPA-receptor specific biogenesis complexes control synaptic transmission and intellectual ability. Nat. Commun. 8:15910. doi: 10.1038/ ncomms 15910

Bredt, D. S., and Nicoll, R. A. (2003). AMPA receptor trafficking at excitatory synapses. Neuron 40, 361-379. doi: 10.1016/S0896-6273(03)00640-8

Cathala, L., Holderith, N. B., Nusser, Z., DiGregorio, D. A, and Cull-Candy, S. G. (2005). Changes in synaptic structure underlie the developmental speeding of AMPA receptor-mediated EPSCs. Nat. Neurosci. 8, 1310-1318. doi: 10.1038/ nn1534

Chen, L., Chetkovich, D. M., Petralia, R. S., Sweeney, N. T., Kawasaki, Y., Wenthold, R. J., et al. (2000). Stargazin regulates synaptic targeting of AMPA receptors by two distinct mechanisms. Nature 408, 936-943. doi: 10.1038/ 35050030

Chen, N., Pandya, N. J., Koopmans, F., Castelo-Szekelv, V., van der Schors, R. C., Smit, A. B., et al. (2014). Interaction proteomics reveals brain region-specific AMPA receptor complexes. J. Proteome Res. 13, 5695-5706. doi: 10.1021/ pr500697b

Coombs, I. D., Soto, D., Zonouzi, M., Renzi, M., Shelley, C., Farrant, M., et al. (2012). Cornichons modify channel properties of recombinant and glial AMPA receptors. J. Neurosci. 32, 9796-9804. doi: 10.1523/JNEUROSCI.0345-12.2012

Encalada, S. E., Szpankowski, L., Xia, C. H., and Goldstein, L. S. (2011). Stable kinesin and dynein assemblies drive the axonal transport of mammalian prion protein vesicles. Cell 144, 551-565. doi: 10.1016/j.cell.2011.01.021

Erlenhardt, N., Yu, H., Abiraman, K., Yamasaki, T., Wadiche, J. I., Tomita, S., et al. (2016). Porcupine controls hippocampal AMPAR levels, composition, and synaptic transmission. Cell Rep. 14, 782-794. doi: 10.1016/j.celrep.2015.12.078

Fado, R., Soto, D., Minano-Molina, A. J., Pozo, M., Carrasco, P., Yefimenko, N., et al. (2015). Novel Regulation of the synthesis of alpha-amino-3-hydroxy-5methyl-4-isoxazolepropionic acid (AMPA) receptor subunit GluA1 by carnitine palmitoyltransferase 1C (CPT1C) in the hippocampus. J. Biol. Chem. 290, 25548-25560. doi: 10.1074/jbc.M115.681064

Farrow, P., Khodosevich, K., Sapir, Y., Schulmann, A., Aslam, M., Stern-Bach, Y., et al. (2015). Auxiliary subunits of the CKAMP family differentially modulate AMPA receptor properties. Elife 4:e09693. doi: 10.7554/eLife.09693

Gill, M. B., Kato, A. S., Roberts, M. F., Yu, H., Wang, H., Tomita, S., et al. (2011). Cornichon-2 modulates AMPA receptor-transmembrane AMPA authors and does not necessarily represent the official views of the National Institutes of Health.

\section{ACKNOWLEDGMENT}

The authors thank Cindy Clark, NIH Library Writing Center, for manuscript editing assistance.

\section{SUPPLEMENTARY MATERIAL}

The Supplementary Material for this article can be found online at: https://www.frontiersin.org/articles/10.3389/fnmol. 2017.00402/full\#supplementary-material

receptor regulatory protein assembly to dictate gating and pharmacology. J. Neurosci. 31, 6928-6938. doi: 10.1523/JNEUROSCI.6271-10.2011

Gill, M. B., Kato, A. S, Wang, H., and Bredt, D. S. (2012). AMPA receptor modulation by cornichon-2 dictated by transmembrane AMPA receptor regulatory protein isoform. Eur. J. Neurosci. 35, 182-194. doi: 10.1111/j.14609568.2011.07948.x

Gratacos-Batlle, E., Yefimenko, N., Cascos-Garcia, H., and Soto, D. (2014). AMPAR interacting protein CPT1C enhances surface expression of GluA1containing receptors. Front. Cell. Neurosci. 8:469. doi: 10.3389/fncel.2014.00469 Greger, I. H., Watson, J. F., and Cull-Candy, S. G. (2017). Structural and functional architecture of AMPA-type glutamate receptors and their auxiliary proteins. Neuron 94, 713-730. doi: 10.1016/j.neuron.2017.04.009

Gu, X., Mao, X., Lussier, M. P., Hutchison, M. A., Zhou, L., Hamra, F. K., et al. (2016a). GSG1L suppresses AMPA receptor-mediated synaptic transmission and uniquely modulates AMPA receptor kinetics in hippocampal neurons. Nat. Commun. 7:10873. doi: 10.1038/ncomms10873

Gu, X., Zhou, L., and Lu, W. (2016b). An NMDA receptor-dependent mechanism underlies inhibitory synapse development. Cell Rep. 14, 471-478. doi: 10.1016/ j.celrep.2015.12.061

Haering, S. C., Tapken, D., Pahl, S., and Hollmann, M. (2014). Auxiliary subunits: shepherding AMPA receptors to the plasma membrane. Membranes 4, 469-490. doi: 10.3390/membranes4030469

Heisler, F. F., Lee, H. K., Gromova, K. V., Pechmann, Y., Schurek, B., Ruschkies, L., et al. (2014). GRIP1 interlinks N-cadherin and AMPA receptors at vesicles to promote combined cargo transport into dendrites. Proc. Natl. Acad. Sci. U.S.A. 111, 5030-5035. doi: 10.1073/pnas.1304301111

Herring, B. E., Shi, Y., Suh, Y. H., Zheng, C. Y., Blankenship, S. M., Roche, K. W., et al. (2013). Cornichon proteins determine the subunit composition of synaptic AMPA receptors. Neuron 77, 1083-1096. doi: 10.1016/j.neuron.2013.01.017

Hoerndli, F. J., Maxfield, D. A., Brockie, P. J., Mellem, J. E., Jensen, E., Wang, R., et al. (2013). Kinesin-1 regulates synaptic strength by mediating the delivery, removal, and redistribution of AMPA receptors. Neuron 80, 1421-1437. doi: 10.1016/j.neuron.2013.10.050

Hoerndli, F. J., Wang, R., Mellem, J. E., Kallarackal, A., Brockie, P. J., Thacker, C., et al. (2015). Neuronal activity and CaMKII regulate kinesin-mediated transport of synaptic AMPARs. Neuron 86, 457-474. doi: 10.1016/j.neuron. 2015.03.011

Huganir, R. L., and Nicoll, R. A. (2013). AMPARs and synaptic plasticity: the last 25 years. Neuron 80, 704-717. doi: 10.1016/j.neuron.2013.10.025

Jackson, A. C., and Nicoll, R. A. (2011). The expanding social network of ionotropic glutamate receptors: TARPs and other transmembrane auxiliary subunits. Neuron 70, 178-199. doi: 10.1016/j.neuron.2011.04.007

Jacobi, E., and von Engelhardt, J. (2017). Diversity in AMPA receptor complexes in the brain. Curr. Opin. Neurobiol. 45, 32-38. doi: 10.1016/j.conb.2017.03.001

Kalashnikova, E., Lorca, R. A., Kaur, I., Barisone, G. A., Li, B., Ishimaru, T., et al. (2010). SynDIG1: an activity-regulated, AMPA- receptor-interacting transmembrane protein that regulates excitatory synapse development. Neuron 65, 80-93. doi: 10.1016/j.neuron.2009.12.021 
Kapitein, L. C., Schlager, M. A., Kuijpers, M., Wulf, P. S., van Spronsen, M., MacKintosh, F. C., et al. (2010). Mixed microtubules steer dynein-driven cargo transport into dendrites. Curr. Biol. 20, 290-299. doi: 10.1016/j.cub.2009.12.052

Kato, A. S., Gill, M. B., Ho, M. T., Yu, H., Tu, Y., Siuda, E. R., et al. (2010). Hippocampal AMPA receptor gating controlled by both TARP and cornichon proteins. Neuron 68, 1082-1096. doi: 10.1016/j.neuron.2010.11.026

Khodosevich, K., Jacobi, E., Farrow, P., Schulmann, A., Rusu, A., Zhang, L., et al. (2014). Coexpressed auxiliary subunits exhibit distinct modulatory profiles on AMPA receptor function. Neuron 83, 601-615. doi: 10.1016/j.neuron.2014. 07.004

Kim, C. H., and Lisman, J. E. (2001). A labile component of AMPA receptormediated synaptic transmission is dependent on microtubule motors, actin, and N-ethylmaleimide-sensitive factor. J. Neurosci. 21, 4188-4194

Klaassen, R. V., Stroeder, J., Coussen, F., Hafner, A. S., Petersen, J. D., Renancio, C., et al. (2016). Shisa6 traps AMPA receptors at postsynaptic sites and prevents their desensitization during synaptic activity. Nat. Commun. 7:10682. doi: $10.1038 /$ ncomms 10682

Li, J., Han, W., Pelkey, K. A., Duan, J., Mao, X., Wang, Y. X., et al. (2017). Molecular dissection of neuroligin 2 and Slitrk3 reveals an essential framework for GABAergic synapse development. Neuron 96, 808-826. doi: 10.1016/j. neuron.2017.10.003

Lu, W., Shi, Y., Jackson, A. C., Bjorgan, K., During, M. J, Sprengel, R., et al. (2009). Subunit composition of synaptic AMPA receptors revealed by a single-cell genetic approach. Neuron 62, 254-268. doi: 10.1016/j.neuron.2009.02.027

Madeo, M., Stewart, M., Sun, Y., Sahir, N., Wiethoff, S., Chandrasekar, I., et al. (2016). Loss-of-function mutations in FRRS1L lead to an epileptic-dyskinetic encephalopathy. Am. J. Hum. Genet. 98, 1249-1255. doi: 10.1016/j.ajhg.2016. 04.008

Malenka, R. C., and Bear, M. F. (2004). LTP and LTD: an embarrassment of riches. Neuron 44, 5-21. doi: 10.1016/j.neuron.2004.09.012

Malinow, R., and Malenka, R. C. (2002). AMPA receptor trafficking and synaptic plasticity. Annu. Rev. Neurosci. 25, 103-126. doi: 10.1146/annurev.neuro.25. 112701.142758

Mao, X., Gu, X., and Lu, W. (2017). GSG1L regulates the strength of AMPA receptor-mediated synaptic transmission but not AMPA receptor kinetics in hippocampal dentate granule neurons. J. Neurophysiol. 117, 28-35. doi: 10.1152/jn.00307.2016

McGee, T. P., Bats, C., Farrant, M., and Cull-Candy, S. G. (2015). Auxiliary subunit GSG1L acts to suppress calcium-permeable AMPA receptor function. J. Neurosci. 35, 16171-16179. doi: 10.1523/JNEUROSCI.2152-15.2015

Rouach, N., Byrd, K., Petralia, R. S., Elias, G. M., Adesnik, H., Tomita, S., et al. (2005). TARP gamma-8 controls hippocampal AMPA receptor number, distribution and synaptic plasticity. Nat. Neurosci. 8, 1525-1533. doi: 10.1038/ nn1551

Schwenk, J., Harmel, N., Brechet, A., Zolles, G., Berkefeld, H., Muller, C. S., et al. (2012). High-resolution proteomics unravel architecture and molecular diversity of native AMPA receptor complexes. Neuron 74, 621-633. doi: 10.1016/j.neuron.2012.03.034
Schwenk, J., Harmel, N., Zolles, G., Bildl, W., Kulik, A., Heimrich, B., et al. (2009). Functional proteomics identify cornichon proteins as auxiliary subunits of AMPA receptors. Science 323, 1313-1319. doi: 10.1126/science.1167852

Setou, M., Seog, D. H., Tanaka, Y., Kanai, Y., Takei, Y., Kawagishi, M., et al. (2002). Glutamate-receptor-interacting protein GRIP1 directly steers kinesin to dendrites. Nature 417, 83-87. doi: 10.1038/nature743

Shaheen, R., Al Tala, S., Ewida, N., Abouelhoda, M., and Alkuraya, F. S. (2016). Epileptic encephalopathy with continuous spike-and-wave during sleep maps to a homozygous truncating mutation in AMPA receptor component FRRS1L. Clin. Genet. 90, 282-283. doi: 10.1111/cge.12796

Shanks, N. F., Savas, J. N., Maruo, T., Cais, O., Hirao, A., Oe, S., et al. (2012). Differences in AMPA and kainate receptor interactomes facilitate identification of AMPA receptor auxiliary subunit GSG1L. Cell Rep. 1, 590-598. doi: 10.1016/ j.celrep.2012.05.004

Shi, Y., Suh, Y. H., Milstein, A. D., Isozaki, K., Schmid, S. M., Roche, K. W., et al. (2010). Functional comparison of the effects of TARPs and cornichons on AMPA receptor trafficking and gating. Proc. Natl. Acad. Sci. U.S.A. 107, 16315-16319. doi: 10.1073/pnas.1011706107

Straub, C., and Tomita, S. (2012). The regulation of glutamate receptor trafficking and function by TARPs and other transmembrane auxiliary subunits. Curr. Opin. Neurobiol. 22, 488-495. doi: 10.1016/j.conb.2011. 09.005

Tomita, S., Chen, L., Kawasaki, Y., Petralia, R. S., Wenthold, R. J., Nicoll, R. A., et al. (2003). Functional studies and distribution define a family of transmembrane AMPA receptor regulatory proteins. J. Cell Biol. 161, 805-816. doi: 10.1083/jcb. 200212116

Tomita, S., Fukata, M., Nicoll, R. A., and Bredt, D. S. (2004). Dynamic interaction of stargazin-like TARPs with cycling AMPA receptors at synapses. Science 303, 1508-1511. doi: 10.1126/science.1090262

von Engelhardt, J., Mack, V., Sprengel, R., Kavenstock, N., Li, K. W., SternBach, Y., et al. (2010). CKAMP44: a brain-specific protein attenuating short-term synaptic plasticity in the dentate gyrus. Science 327, 1518-1522. doi: $10.1126 /$ science. 1184178

Wei, M., Zhang, J., Jia, M., Yang, C., Pan, Y., Li, S., et al. (2016). Alpha/betahydrolase domain-containing 6 (ABHD6) negatively regulates the surface delivery and synaptic function of AMPA receptors. Proc. Natl. Acad. Sci. U.S.A. 113, E2695-E27704. doi: 10.1073/pnas.1524589113

Conflict of Interest Statement: The authors declare that the research was conducted in the absence of any commercial or financial relationships that could be construed as a potential conflict of interest.

Copyright (C) 2017 Han, Wang, Li, Zhang and Lu. This is an open-access article distributed under the terms of the Creative Commons Attribution License (CC BY). The use, distribution or reproduction in other forums is permitted, provided the original author(s) or licensor are credited and that the original publication in this journal is cited, in accordance with accepted academic practice. No use, distribution or reproduction is permitted which does not comply with these terms. 